\title{
Melatonin Therapy Prevents Programmed Hypertension and Nitric Oxide Deficiency in Offspring Exposed to Maternal Caloric Restriction
}

\author{
You-Lin Tain, ${ }^{1,2}$ Li-Tung Huang, ${ }^{1,3}$ Chien-Ning Hsu, ${ }^{4,5}$ and Chien-Te Lee ${ }^{6}$ \\ ${ }^{1}$ Department of Pediatrics, Kaohsiung Chang Gung Memorial Hospital and College of Medicine, Chang Gung University, \\ Kaohsiung 833, Taiwan \\ ${ }^{2}$ Center for Translational Research in Biomedical Sciences, Kaohsiung Chang Gung Memorial Hospital and College of Medicine, \\ Chang Gung University, Kaohsiung 833, Taiwan \\ ${ }^{3}$ Department of Traditional Chinese Medicine, Chang Gung University, Linkou 333, Taiwan \\ ${ }^{4}$ Department of Pharmacy, Kaohsiung Chang Gung Memorial Hospital and College of Medicine, Chang Gung University, \\ Kaohsiung 833, Taiwan \\ ${ }^{5}$ Graduate Institute of Clinical Pharmacy, College of Pharmacy, Kaohsiung Medical University, Kaohsiung 833, Taiwan \\ ${ }^{6}$ Division of Nephrology, Department of Internal Medicine, Kaohsiung Chang Gung Memorial Hospital and College of Medicine, \\ Chang Gung University, Kaohsiung 833, Taiwan
}

Correspondence should be addressed to Chien-Te Lee; chientel@gmail.com

Received 6 January 2014; Revised 18 March 2014; Accepted 28 March 2014; Published 22 April 2014

Academic Editor: Jean-Claude Lavoie

Copyright (C) 2014 You-Lin Tain et al. This is an open access article distributed under the Creative Commons Attribution License, which permits unrestricted use, distribution, and reproduction in any medium, provided the original work is properly cited.

\begin{abstract}
Nitric oxide (NO) deficiency is involved in the development of hypertension, a condition that can originate early in life. We examined whether NO deficiency contributed to programmed hypertension in offspring from mothers with calorie-restricted diets and whether melatonin therapy prevented this process. We examined 3-month-old male rat offspring from four maternal groups: untreated controls, $50 \%$ calorie-restricted (CR) rats, controls treated with melatonin $(0.01 \%$ in drinking water), and CR rats treated with melatonin $(\mathrm{CR}+\mathrm{M})$. The effect of melatonin on nephrogenesis was analyzed using next-generation sequencing. The CR group developed hypertension associated with elevated plasma asymmetric dimethylarginine (ADMA, a nitric oxide synthase inhibitor), decreased L-arginine, decreased L-arginine-to-ADMA ratio (AAR), and decreased renal NO production. Maternal melatonin treatment prevented these effects. Melatonin prevented CR-induced renin and prorenin receptor expression. Renal angiotensinconverting enzyme 2 protein levels in the $\mathrm{M}$ and $\mathrm{CR}+\mathrm{M}$ groups were also significantly increased by melatonin therapy. Maternal melatonin therapy had long-term epigenetic effects on global gene expression in the kidneys of offspring. Conclusively, we attributed these protective effects of melatonin on CR-induced programmed hypertension to the reduction of plasma ADMA, restoration of plasma AAR, increase of renal NO level, alteration of renin-angiotensin system, and epigenetic changes in numerous genes.
\end{abstract}

\section{Introduction}

Hypertension might originate during early life. Maternal malnutrition can impair development, resulting in intrauterine growth restriction (IUGR), permanent structural changes, and disrupted physiological function - a phenomenon called "developmental programming" [1]. In the kidneys of both humans and experimental models, developmental programming reduces nephron numbers, alters the renin-angiotensin system (RAS), and impairs natriuresis, leading to adult kidney disease and hypertension [2-5].

A number of hypotheses have been proposed to explain the developmental programming phenomenon [6]. Oxidative stress is proposed as the underlying link between developmental programing and elevated risks of hypertension and kidney disease in adulthood [7, 8]. Asymmetric dimethylarginine (ADMA), an endogenous inhibitor of $\mathrm{NO}$ synthase (NOS), causes oxidative stress and is involved 
in the development of hypertension [9]. Our recent work demonstrated that an impaired ADMA-NO pathway and low nephron numbers are associated with programmed hypertension in the adult offspring of malnourished or diabetic mothers [10,11]. Reduced nephron numbers impaired renal tubular sodium reabsorption, and the altered RAS components disrupted sodium retention, ultimately increasing blood pressure (BP) and inducing kidney damage. Histone deacetylases (HDACs) repress gene expression, a mechanism of epigenetic control that is involved in developmental programming. Class I HDACs are critical in nephrogenesis, particularly HDAC1-3 that are highly expressed in nephron precursors [12]. HDACs also play an important role in regulating RAS components during nephrogenesis [13]. These observations suggest that these mechanisms jointly lead to the development of hypertension and kidney disease.

Melatonin, an indoleamine produced from the pineal gland, is an antioxidant and free radical scavenger [14]. Experimental and human studies indicate that melatonin can regulate BP $[10,11]$. We recently found that melatonin can prevent oxidative stress and hypertension concurrently in young spontaneously hypertensive rats (SHR) [15]. Emerging evidence supports novel roles of melatonin in epigenetic modulation through the regulation of HDACs $[16,17]$. Thus, we examined whether melatonin prevented programmed hypertension in offspring exposed to maternal caloric restriction through reduction of oxidative stress, alteration of the RAS pathway, and modulation of HDACs. Moreover, we identified melatonin-induced gene changes during nephrogenesis and determined whether melatonin treatment induced global changes in biological processes by using next-generation sequencing.

\section{Material and Methods}

2.1. Animal Models. This study was carried out in strict accordance with the recommendations of the Guide for the Care and Use of Laboratory Animals of the National Institutes of Health. The protocol was approved by the Institutional Animal Care and Use Committee of the Kaohsiung Chang Gung Memorial Hospital. Virgin Sprague-Dawley (SD) rats (12-16 weeks old) were obtained from BioLASCO Taiwan Co., Ltd. (Taipei, Taiwan), and were housed and maintained in a facility accredited by the Association for Assessment and Accreditation of Laboratory Animal Care International. Male $\mathrm{SD}$ rats were caged with individual females until mating was confirmed. Calorie-restricted (CR) maternal rats received $11 \mathrm{~g} / \mathrm{d}$ of a standard chow from day 11 of pregnancy until the day of delivery (day 23) and $20 \mathrm{~g} / \mathrm{d}$ during the entire lactation period [10]. A subset of CR mothers was treated for the duration of the pregnancy with $0.01 \%$ melatonin dissolved in drinking water $(\mathrm{CR}+\mathrm{M}, n=8)$. The control group $(n=8)$ mothers had free access to standard rat chow. As another control, maternal rats were allowed free access to standard rat chow and were treated with $0.01 \%$ melatonin in drinking water $(\mathrm{M}, n=10)$. After birth, each litter was left with the mother until weaning; pups were not weighed at birth to prevent maternal rejection. Male offspring, selected at random from each litter, were used in all subsequent experiments. In rats, nephrogenesis occurs predominantly from late gestation to 1-2 weeks postnatum and litters were typically weaned by postnatal week 3 . Thus, melatonin was administered to mother rats for a total period of 6 weeks to cover the entire period of nephrogenesis. The dose of melatonin used was based on our previous study [15]. Water bottles were covered with aluminum foil to protect them from light. BP was measured in conscious rats by using an indirect tail-cuff method (BP-2000, Visitech Systems, Inc., Apex, NC, USA) after they had been systematically trained [10]. Three stable consecutive measures were taken and averaged. All offspring were sacrificed at 12 weeks of age and heparinized blood samples were collected. Kidneys were harvested after perfusion with PBS, divided into cortex and medulla regions, and snap-frozen. The activity of dimethylarginine dimethylaminohydrolase (DDAH), an ADMA-metabolizing enzyme, was measured using a colorimetric assay. The assay determined the rate of $\mathrm{L}$-citrulline production and we performed the assay as previously described [18].

\subsection{High-Performance Liquid Chromatography (HPLC).} Plasma and kidney L-arginine, L-citrulline, ADMA, and symmetric dimethylarginine (SDMA, a stereoisomer of ADMA) levels were measured using HPLC (HP series 1100, Agilent Technologies, Inc., Santa Clara, CA, USA) with the OPA-3MPA derivatization reagent as we described previously [10]. Standards contained L-arginine, L-citrulline, ADMA, and SDMA in the range of $1-100 \mu \mathrm{M}, 1-100 \mu \mathrm{M}, 0.5-5 \mu \mathrm{M}$, and $0.5-5 \mu \mathrm{M}$, respectively. The recovery rate was between 90 and $105 \%$. The tissue concentration was factored for protein concentration, which was represented as $\mu \mathrm{mol} / \mathrm{mg}$ protein. Plasma and urine creatinine ( $\mathrm{Cr}$ ) levels were analyzed by HPLC as described previously [10]. The creatinine clearance (CCr) was calculated by dividing the total amount of $\mathrm{Cr}$ excreted in urine by the $\mathrm{Cr}$ concentration in plasma. $\mathrm{CCr}$ values were normalized with respect to body weight.

\subsection{Electron Paramagnetic Resonance (EPR). Superoxide} production was measured by EPR spectroscopy using a 1hydroxy-3-carboxypyrrolidine $(\mathrm{CPH})$ hydroxylamine spin probe, as we previously described [11]. The EPR spectra were recorded using an EMX Plus EPR spectrometer (Bruker BioSpin, Rheinstetten, Germany) equipped with an EMXm40X microwave bridge operating at $9.87 \mathrm{GHz}$. NO was detected by EPR using N-methyl-D-glucamine dithiocarbamate (MGD) spin probe and $\mathrm{FeSO}_{4}$, as previously described [11]. The EPR spectra were recorded using an EMX Plus EPR spectrometer (Bruker BioSpin) equipped with an EMXm40X microwave bridge operating at $3.16 \mathrm{GHz}$.

2.4. Metanephros Organ Culture. Metanephros organ culture was performed as we described previously [11]. Briefly, $\mathrm{SD}$ female rats of known mating date were anesthetized and laparotomized. Fetuses were aseptically removed, and metanephroi from fetuses at embryonic day 14 (E14) were collected and freed of exogenous tissue. Explants were placed onto a Steritop filter unit (Millipore, Billerica, MA, USA) 
floating on a defined serum-free medium and incubated for $6 \mathrm{~d}$ in $35 \mathrm{~mm}$ Petri dishes at $37^{\circ} \mathrm{C}$ in a humidified incubator $\left(5 \% \mathrm{CO}_{2}\right)$. The defined medium was composed of Eagle's Minimum Essential Medium containing 10\% (v/v) fetal calf serum, 100 units $/ \mathrm{mL}$ penicillin, and $100 \mu \mathrm{g} / \mathrm{mL}$ streptomycin. All of these reagents were obtained from Sigma (St. Louis, MO, USA). The culture medium was changed daily, and no antibiotic or fungicide was present throughout the experiment. Fresh aliquots of each culture medium additive were used for each metanephros culture. The medium was changed daily. Metanephroi were treated with melatonin $(1 \mu \mathrm{M}$ and $1 \mathrm{mM}$ ) and harvested after $6 \mathrm{~d}$ for real-time polymerase chain reaction.

\subsection{Quantitative Real-Time Polymerase Chain Reaction} (PCR). RNA was extracted as described previously [10]. Twostep quantitative real-time PCR was conducted using the QuantiTect SYBR Green PCR Kit (Qiagen, Valencia, CA, USA) and the iCycler iQ Multicolor Real-Time PCR Detection System (Bio-Rad, Hercules, CA, USA). Nephron deficit was assessed by changes in the expression factors known to be involved in branching morphogenesis (BMP4, FGF2, and PAX2) and apoptosis (p53 and Bax). Several components of the RAS were analyzed including renin, prorenin receptor (PRR), angiotensinogen (AGT), angiotensin-converting enzyme (ACE), ACE2, angiotensin II type 1 (AT1R) and 2 receptor (AT2R), and angiotensin (1-7) MAS receptor. Class I HDACs, HDAC-1, $-2,-3$, and -8 , were also examined. We used $18 \mathrm{~S}$ rRNA (r18S) as a reference. Primers were designed using GeneTool Software (BioTools, Edmonton, Alberta, Canada) (Table 1). All samples were run in duplicate. To quantify the relative gene expression, the comparative threshold cycle $\left(\mathrm{C}_{\mathrm{T}}\right)$ method was employed. For each sample, the average $C_{T}$ value was subtracted from the corresponding average r18S value, calculating the $\Delta \mathrm{C}_{\mathrm{T}} . \Delta \Delta \mathrm{C}_{\mathrm{T}}$ was calculated by subtracting the average control $\Delta \mathrm{C}_{\mathrm{T}}$ value from the average experimental $\Delta \mathrm{C}_{\mathrm{T}}$. The fold-increase of the experimental sample relative to the control was calculated using the formula $2^{-\Delta \Delta \mathrm{C}_{\mathrm{T}}}$.

2.6. Western Blot. Western blot analysis was performed as previously described [10]. We used the following antibodies from Santa Cruz Biotechnology (Santa Cruz, CA, USA): rabbit polyclonal anti-rat PRR (1:500, overnight incubation), rabbit anti-rat ACE2 (1:1000, overnight incubation), rabbit anti-rat AT1R ( $1: 250$, overnight incubation), rabbit anti-rat AT2R ( $1: 250$, overnight incubation), and rabbit anti-rat MAS (1:1000, overnight incubation; Santa Cruz Biotechnology). The bands of interest were visualized using enhanced chemiluminescence reagent (PerkinElmer, Waltham, MA, USA) and quantified by densitometry (Quantity One Analysis software, Bio-Rad). Band density was calculated as the integrated optical density (IOD) minus the background value. The density of Ponceau red staining (PonS) was used to correct for variations in total protein loading. Protein abundance was calculated as IOD/PonS.

2.7. Next-Generation Sequencing and Analysis. In rats, nephrogenesis occurs predominantly from late gestation to 7-10 days postnatum. Thus, offspring from the control and $\mathrm{M}$ groups were sacrificed at 1 week of age. Kidneys were isolated and snap-frozen for whole-genome RNA next-generation sequencing (RNA-seq), performed by Welgene Biotech Co., Ltd. (Taipei, Taiwan). Purified RNA was quantified at 260 $\mathrm{nm}\left(\mathrm{OD}_{600}\right)$ by using ND-1000 spectrophotometer (Nanodrop Technology, Wilmington, DE, USA) and analyzed using a Bioanalyzer 2100 (Agilent Technology) with RNA 6000 LabChip kit (Agilent Technologies). All procedures were performed according to the Illumina protocol. For all samples, library construction was performed using the TruSeq RNA Sample Prep Kit v2 for 160 bp (single-end) sequencing and the Solexa platform. The sequence was directly determined by sequencing-by-synthesis technology using the TruSeq SBS Kit. Raw sequences were obtained using the Illumina GA Pipeline software CASAVA v1.8, which was expected to generate 10 million reads per sample. Quantification for gene expression was calculated as reads per kilobase of exon per million mapped reads [19]. For differential expression analysis, Cufflink v 2.1.1 and CummeRbund v 2.0.0 were used to perform statistical analyses of the gene expression profiles. The reference genome and gene annotations were retrieved from the Ensembl database (http://asia.ensembl.org/index.html). Gene ontology analysis for significant genes was performed using KEGG (http://www.genome.jp/kegg/) and NIH DAVID Bioinformatics Resources 6.7 (http://david.abcc.ncifcrf.gov/) to identify regulated biological themes.

2.8. Statistical Analysis. TheShapiro-Wilk normality test was used to determine which data were normally distributed. Normally distributed data are given as mean \pm standard error of the mean. For most parameters, statistical analysis was performed using one-way analysis of variance (ANOVA) and Tukey's post hoc test for multiple comparisons. BP was analyzed by two-way repeated-measures ANOVA and Tukey's post hoc test. A $P$ value $<0.05$ was considered statistically significant. Analyses were performed using the Statistical Package for the Social Sciences (SPSS) software (Chicago, IL, USA).

\section{Results}

3.1. The Effects of Melatonin on Morphological and Biochemical Values in CR Rats. Litter sizes were not significantly altered by caloric restriction in the mother rat or by melatonin treatment. The amounts of water intake and urine output were not significantly different in the control and CR groups. Male pup mortality rates did not differ between the four groups analyzed. As shown in Table 2, the CR and M groups had lower and higher body weight (BW) than the control at 12 weeks of age, respectively, whereas the $\mathrm{CR}+\mathrm{M}$ group had an intermediate BW. Kidney weight and kidney weightto-BW ratio did not differ between the control and CR groups. Melatonin significantly increased kidney weight and kidney weight-to-BW ratio in the $M$ and $C R+M$ groups. Although heart weight was not different between control and CR groups, the heart weight-to-BW ratio was greater 
in the CR group. Melatonin caused increased heart weight and heart weight-to-BW ratio in the $\mathrm{M}$ group, but not in the $\mathrm{CR}+\mathrm{M}$ group. $\mathrm{CR}$ increased systolic and diastolic BP and mean arterial pressure at 12 weeks of age. Melatonin therapy prevented these effects of CR. In addition, melatonin therapy reduced diastolic BP and mean arterial pressure in the M group compared to the control. As shown in Figure 1, mean arterial pressure was similar in the four groups at 4 weeks of age. By 8 weeks of age, mean arterial pressure had increased in the CR group relative to controls. A significant reduction in mean arterial pressure was measured in the $\mathrm{M}$ and $\mathrm{CR}+\mathrm{M}$ groups versus the control at 8 and 12 weeks of age. In contrast, plasma creatinine level did not differ between the four groups. These data demonstrated that CR induced programmed hypertension but had no effect on renal function on 12-week-old offspring.

3.2. The Effects of Melatonin on L-Arginine, L-Citrulline, and Dimethylarginine Levels. As shown in Table 3, plasma levels of ADMA and SDMA were elevated nearly $70 \%$ and $150 \%$ following maternal $\mathrm{CR}$, respectively. In contrast, the L-arginine levels and L-arginine-to-ADMA ratio were decreased by $30 \%$ and $55 \%$, respectively. Melatonin treatment significantly increased $\mathrm{L}$-arginine levels and $\mathrm{L}$-arginine-to-ADMA ratio, but decreased ADMA and SDMA levels in the CR + M group. In the kidney, levels of L-citrulline, $\mathrm{L}$-arginine, ADMA, and SDMA did not differ between the four groups. However, renal $\mathrm{L}$-arginine-to-ADMA ratio was higher in the $\mathrm{CR}+\mathrm{M}$ group versus the $\mathrm{M}$ group. We next analyzed superoxide and NO production in the kidney by using EPR. We found no difference in renal superoxide level among the four groups (control: $745 \pm 28$, CR: $823 \pm 107$, M: $665 \pm 35$, CR + M: $757 \pm 42$ arbitrary units; $P>0.05$ ). CR significantly reduced renal NO levels, but not in the presence of melatonin (control: $412 \pm 43$, CR: $284 \pm 18$, M: $308 \pm 34$, CR + M: $414 \pm 55$ arbitrary units; control versus $\mathrm{CR}, P<0.05$; CR versus $\mathrm{CR}+\mathrm{M}, P<0.05$ ).

\subsection{The Effects of Melatonin on the ADMA Pathway. Next, we} examined the expression/activity of proteins involved in the ADMA pathway. We found that renal level of protein arginine $\mathrm{N}$-methyltransferase 1 (PRMT-1), an ADMA-synthesizing enzyme, was significantly lower in the $\mathrm{M}$ and $\mathrm{CR}+\mathrm{M}$ groups than that in control and CR groups (Figure 2(b)). However, protein levels of DDAH-1 and -2, ADMA-metabolizing enzymes, in the kidney were not different between the four groups (Figures 2(c) and 2(d)). We found that renal DDAH activity did not differ between control and CR groups (Figure 2(e)). However, melatonin increased renal DDAH activity in both the $\mathrm{M}$ and $\mathrm{CR}+\mathrm{M}$ groups. Thus, we speculate that the increase of systemic ADMA observed with CR is due to excessive synthesis or decreased metabolism in extrarenal tissues. On the other hand, the reduced plasma ADMA levels in response to melatonin might be due to decreased ADMA synthesis and increased ADMA breakdown in the kidney.

3.4. The Effects of Melatonin on Nephrogenesis. We investigated whether changes in nephrogenesis- or apoptosisrelated gene expression were associated with CR-induced

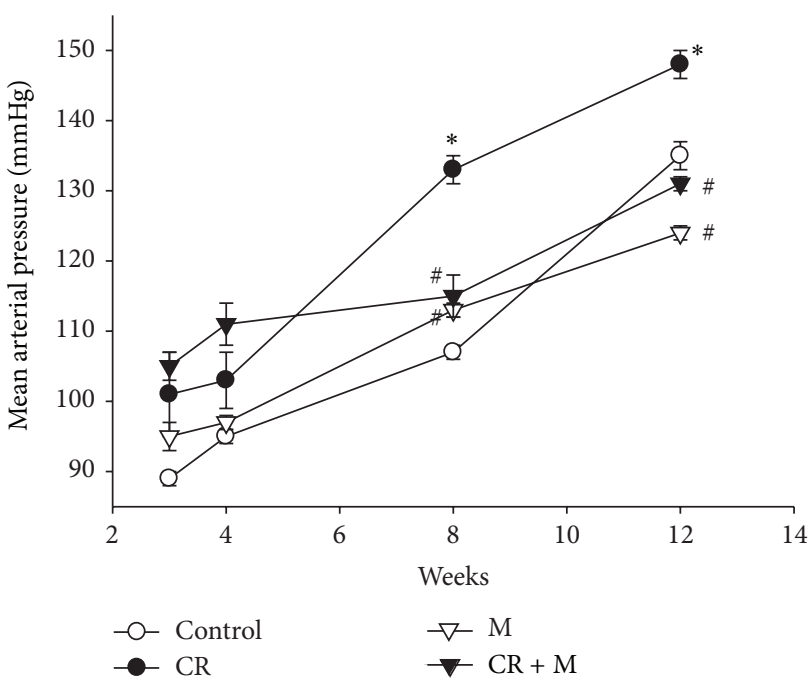

FIGURE 1: Effect of melatonin and caloric restriction (CR) on mean arterial pressure in male offspring at 12 weeks of age. ${ }^{*} P<0.05$ versus control; ${ }^{\#} P<0.05$ versus $C R$.

reduced nephron numbers, as we found previously [10]. Consistent with our previous report [10], renal expression of p53 and the proapoptotic factor Bax did not differ between the control and CR groups (Figure 3(a)). Similarly, growth factors BMP4 and FGF2 were unaltered by CR or melatonin in the kidney. However, melatonin significantly increased the expression of the transcriptional activator PAX2 in $\mathrm{CR}+\mathrm{M}$ group compared to controls (Figure 3(a)).

3.5. The Effects of Melatonin on Sodium Transporters, RAS, and HDACs. Next, we evaluated two critical pathways involved in hypertension, sodium transporters and RAS components. We found that CR upregulated sodium-hydrogen exchanger 3 (NHE3) expression in the kidney (Figure 3(b)). The increase in renal NHE3 expression was not prevented by melatonin therapy. CR had no effect on the expression of RAS genes in the kidney, including renin, PRR, AGT, ACE, ACE2, AT1R, AT2R, and MAS (Figure 3(c)). Melatonin treatment, on the other hand, upregulated renal expression of renin, PRR, and ACE2 in the CR + M group compared to the control. Because melatonin therapy prevented the elevation of BP in offspring exposed to maternal $\mathrm{CR}$, our data suggested that the antihypertensive effect of melatonin was related to renin, PRR, and ACE2 expression in the CR model. We found that CR did not alter renal expression of class I HDACs in the CR versus control group (Figure 3(d)). However, melatonin therapy increased HDAC-2, -3 , and -8 expression in the kidney.

We analyzed the renal protein levels of PRR, ACE2, AT1R, AT2R, and MAS. Melatonin therapy significantly increased renal PRR and ACE2 protein levels in the $\mathrm{M}$ and $\mathrm{CR}+\mathrm{M}$ group compared with the control and CR groups (Figures 4(b) and 4(c)). We observed that renal AT1R, AT2R, and MAS protein levels did not differ among the four groups (Figures $4(d)-4(f))$. 
TABLE 1: PCR primers sequences.

\begin{tabular}{|c|c|c|}
\hline Gene & Forward & Reverse \\
\hline Bax & 5 ttgctgatggcaacttcaactg 3 & 5 ctttagtgcacagggccttgag 3 \\
\hline P53 & 5 catgagcgttgctctgatg 3 & 5 cagatactcagcatacggatttcc 3 \\
\hline PAX2 & 5 gagactcccagagtggtgtg 3 & 5 cattcccetgttctgatttg 3 \\
\hline FGF2 & 5 ccagttggtatgtggcactg 3 & 5 cagggaagggtttgacaaga 3 \\
\hline BMP4 & 5 gacttcgaggcgacacttctg 3 & 5 agccggtaaagatccctcatg 3 \\
\hline Renin & 5 aacattaccagggcaactttcact 3 & 5 accccettcatggtgatctg 3 \\
\hline Prorenin receptor & 5 gaggcagtgaccctcaacat 3 & 5 ccctcctcacacaacaaggt 3 \\
\hline Angiotensinogen & 5 gcccaggtcgcgatgat 3 & 5 tgtacaagatgctgagtgaggcaa 3 \\
\hline ACE & 5 caccggcaaggtctgctt 3 & 5 cttggcatagtttcgtgaggaa 3 \\
\hline ACE2 & 5 accettcttacatcagccctactg 3 & 5 tgtccaaaacctaccccacatat 3 \\
\hline AT1R & 5 gctgggcaacgagtttgtct 3 & 5 cagtccttcagctggatcttca 3 \\
\hline AT2R & 5 caatctggctgtggctgactt 3 & 5 tgcacatcacaggtccaaaga 3 \\
\hline MAS & 5 catctctcctctcggctttgtg 3 & 5 cctcatccggaagcaaagg 3 \\
\hline HDAC-1 & 5 gaactggggacctacggg 3 & 5 gctcttgacaaattccacacac 3 \\
\hline HDAC-2 & 5 agttgcccttgattgtgaga 3 & 5 ccactgttgtccttggatttat 3 \\
\hline HDAC-3 & 5 tgatgaccagagttacaagcac 3 & 5 gggcaacatttc ggacag 3 \\
\hline HDAC-8 & 5 gctacccccggtttatatttacag 3 & 5 ttcgatcagagagtgaaccatactg 3 \\
\hline R18S & 5 gccgcggtaattccagctcca 3 & 5 cccgcccgctcccaagatc 3 \\
\hline
\end{tabular}

TABLE 2: Morphological and biochemical values in different experimental groups.

\begin{tabular}{|c|c|c|c|c|}
\hline & $\begin{array}{c}\text { Control } \\
n=8\end{array}$ & $\begin{array}{c}\text { CR } \\
n=8\end{array}$ & $\begin{array}{c}\mathrm{M} \\
n=10\end{array}$ & $\begin{array}{c}\mathrm{CR}+\mathrm{M} \\
n=8\end{array}$ \\
\hline Mortality & $10 \%$ & $0 \%$ & $0 \%$ & $0 \%$ \\
\hline Body weight (BW) (g) & $435 \pm 14$ & $356 \pm 4^{*}$ & $489 \pm 8^{* \#}$ & $370 \pm 9^{* \$}$ \\
\hline Left kidney weight (g) & $1.22 \pm 0.06$ & $1.01 \pm 0.02$ & $1.97 \pm 0.05^{* \#}$ & $1.48 \pm 0.03^{\# \$}$ \\
\hline Left kidney weight/100g BW & $0.28 \pm 0.01$ & $0.28 \pm 0.01$ & $0.4 \pm 0.01^{* \#}$ & $0.4 \pm 0.01^{* \#}$ \\
\hline Heart weight $(\mathrm{g})$ & $1.23 \pm 0.05$ & $1.24 \pm 0.02$ & $1.63 \pm 0.01^{* \#}$ & $1.16 \pm 0.05^{\$}$ \\
\hline Heart weight/100 g BW & $0.28 \pm 0.01$ & $0.35 \pm 0.01^{*}$ & $0.35 \pm 0.01^{*}$ & $0.31 \pm 0.01$ \\
\hline Systolic blood pressure $(\mathrm{mmHg})$ & $162 \pm 2$ & $180 \pm 2^{*}$ & $155 \pm 1^{\#}$ & $166 \pm 1^{\$}$ \\
\hline Diastolic blood pressure $(\mathrm{mmHg})$ & $122 \pm 2$ & $134 \pm 3^{*}$ & $108 \pm 2^{* \#}$ & $113 \pm 1^{* \#}$ \\
\hline Mean arterial pressure $(\mathrm{mmHg})$ & $135 \pm 2$ & $149 \pm 2^{*}$ & $124 \pm 1^{* \#}$ & $131 \pm 1^{\# \$}$ \\
\hline $\mathrm{CCr}, \mathrm{mL} \cdot \mathrm{min}^{-1} \cdot \mathrm{kg}$ body weight ${ }^{-1}$ & $9.12 \pm 3.45$ & $8.5 \pm 3.0$ & $7.34 \pm 2.32$ & $7.81 \pm 2.76$ \\
\hline
\end{tabular}

CCr: clearance of creatinine; ${ }^{*} P<0.05$ versus control; ${ }^{\#} P<0.05$ versus $C R ;{ }^{\$} P<0.05$ versus $M$.

TABLE 3: L-Citrulline, L-arginine, and dimethylarginine levels in the plasma and kidney.

\begin{tabular}{|c|c|c|c|c|}
\hline & Control & $\mathrm{CR}$ & $\mathrm{M}$ & $\mathrm{CR}+\mathrm{M}$ \\
\hline \multicolumn{5}{|l|}{ Plasma $(\mu \mathrm{mol})$} \\
\hline L-Citrulline & $50 \pm 4.1$ & $61 \pm 3.6$ & $59.3 \pm 5.1$ & $55.8 \pm 6.9$ \\
\hline L-Arginine & $121.1 \pm 14$ & $84.4 \pm 2.4^{*}$ & $113.6 \pm 8.7^{\#}$ & $112.8 \pm 13.6^{\#}$ \\
\hline ADMA & $1.31 \pm 0.1$ & $2.21 \pm 0.18^{*}$ & $1.18 \pm 0.06^{\#}$ & $1.08 \pm 0.12^{\#}$ \\
\hline SDMA & $0.66 \pm 0.04$ & $1.62 \pm 0.27^{*}$ & $0.97 \pm 0.09^{\#}$ & $0.92 \pm 0.08^{\#}$ \\
\hline L-Arginine-to-ADMA ratio & $92 \pm 8$ & $40 \pm 4^{*}$ & $98 \pm 10^{\#}$ & $105 \pm 6^{\#}$ \\
\hline \multicolumn{5}{|l|}{ Kidney ( $\mu \mathrm{mol} / \mathrm{mg}$ protein $)$} \\
\hline L-Citrulline & $52.5 \pm 8.6$ & $53.1 \pm 4.6$ & $97.6 \pm 8.4$ & $68.8 \pm 12.4$ \\
\hline L-Arginine & $425 \pm 62.3$ & $552.9 \pm 58.9$ & $522.8 \pm 61.6$ & $488.1 \pm 56$ \\
\hline ADMA & $5.09 \pm 0.88$ & $6.33 \pm 0.71$ & $6.72 \pm 1.03$ & $4.84 \pm 0.61$ \\
\hline SDMA & $4.3 \pm 0.65$ & $5.3 \pm 0.51$ & $5.57 \pm 0.79$ & $4.59 \pm 0.73$ \\
\hline L-Arginine-to-ADMA ratio & $86 \pm 4$ & $89 \pm 5$ & $80 \pm 4$ & $103 \pm 8^{\$}$ \\
\hline
\end{tabular}

${ }^{*} P<0.05$ versus control; ${ }^{\#} P<0.05$ versus $\mathrm{CR} ;{ }^{\$} P<0.05$ versus $\mathrm{M}$. 


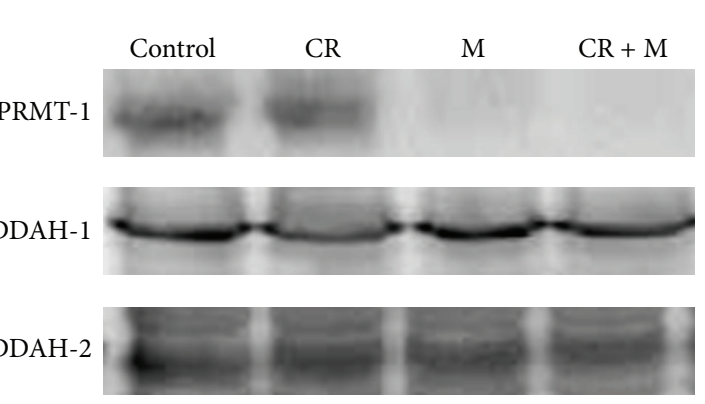

(a)

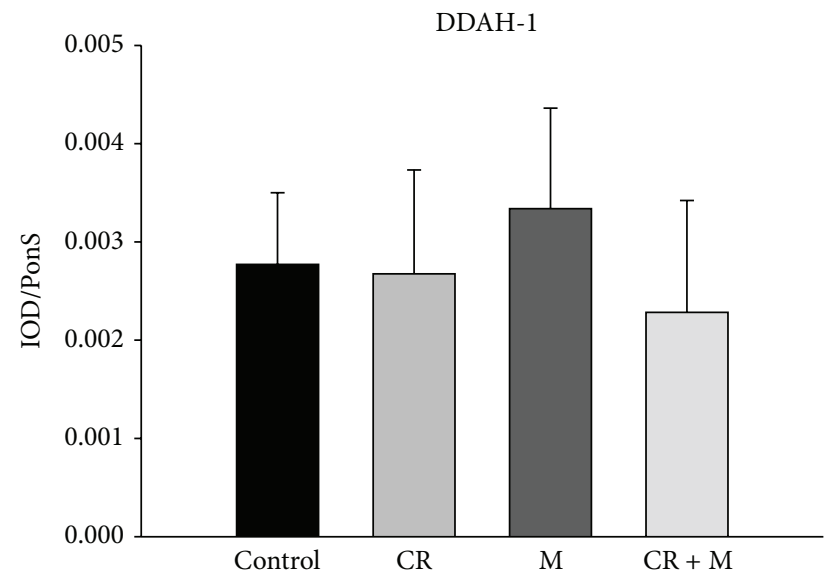

(c)

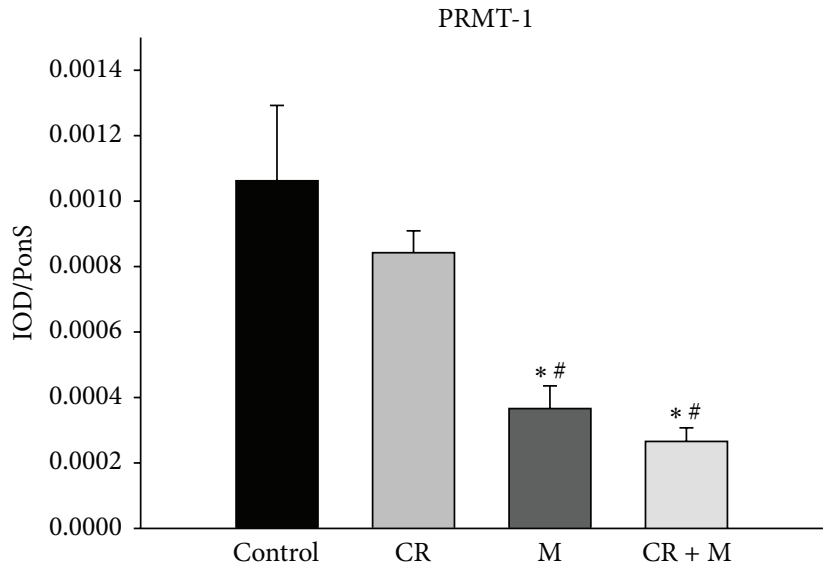

(b)

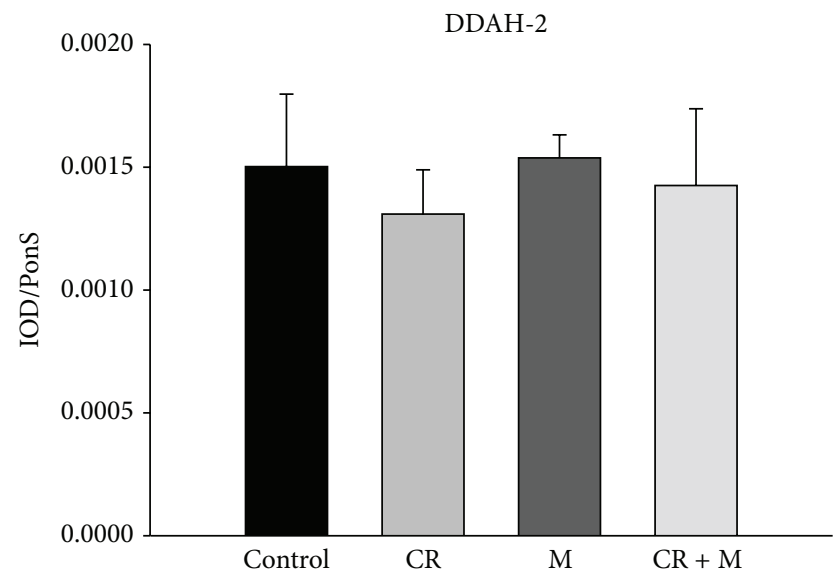

(d)

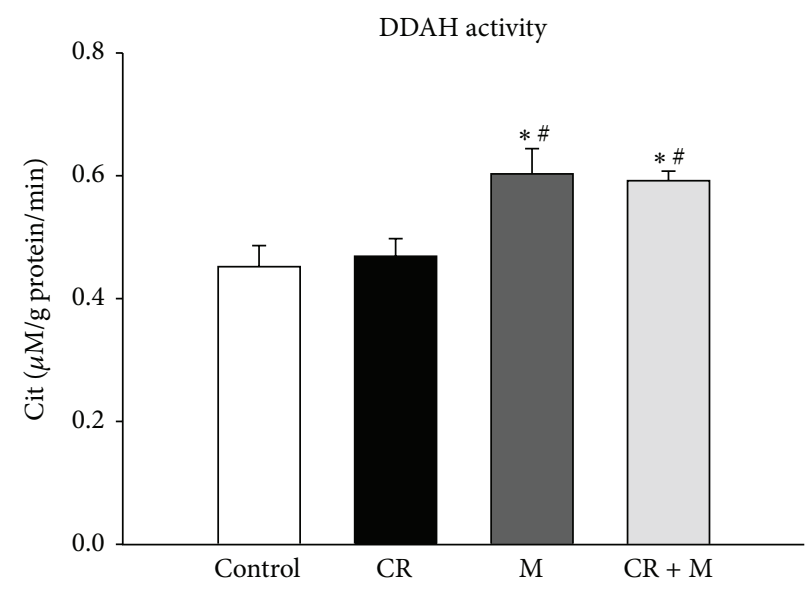

(e)

FIGURE 2: Representative western blots (a) showing protein arginine methyltransferase 1 (PRMT-1; 42 kDa), dimethylarginine dimethylaminohydrolase 1 (DDAH-1; 34 kDa), and DDAH-2 $(\sim 30 \mathrm{kDa})$ bands in CR offspring at 12 weeks of age. Relative abundance of renal cortical (b) PRMT-1, (c) DDAH-1, and (d) DDAH-2. (e) Effect of melatonin and CR on renal DDAH activity in male offspring at 12 weeks of age. ${ }^{*} P<0.05$ versus control; ${ }^{*} P<0.05$ versus $\mathrm{CR}$. 


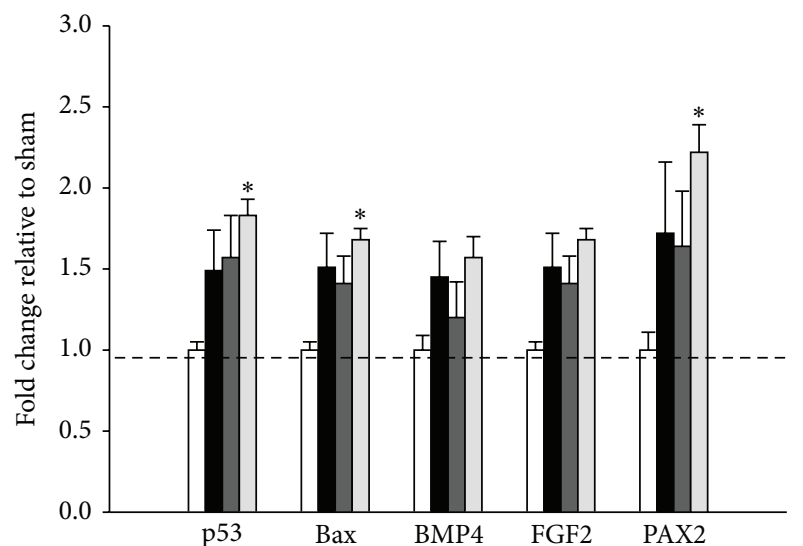

(a)

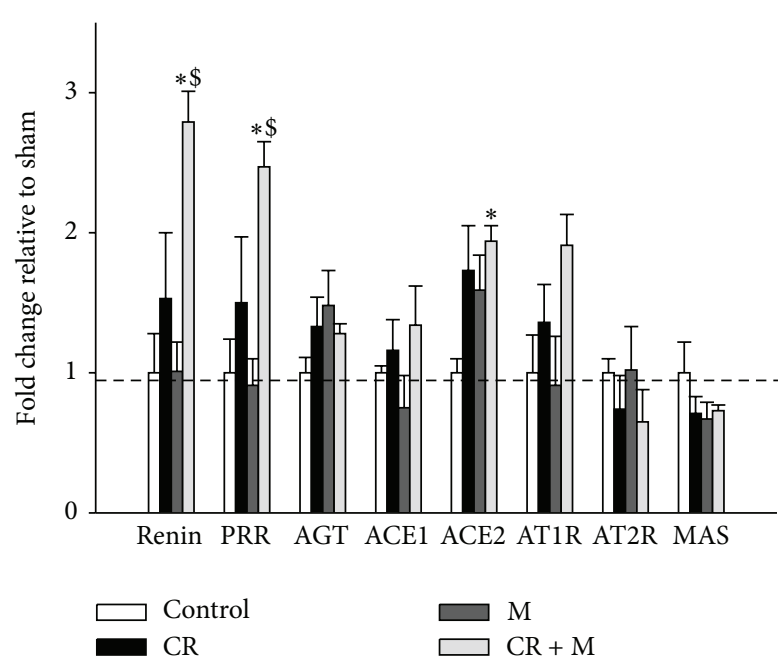

(c)

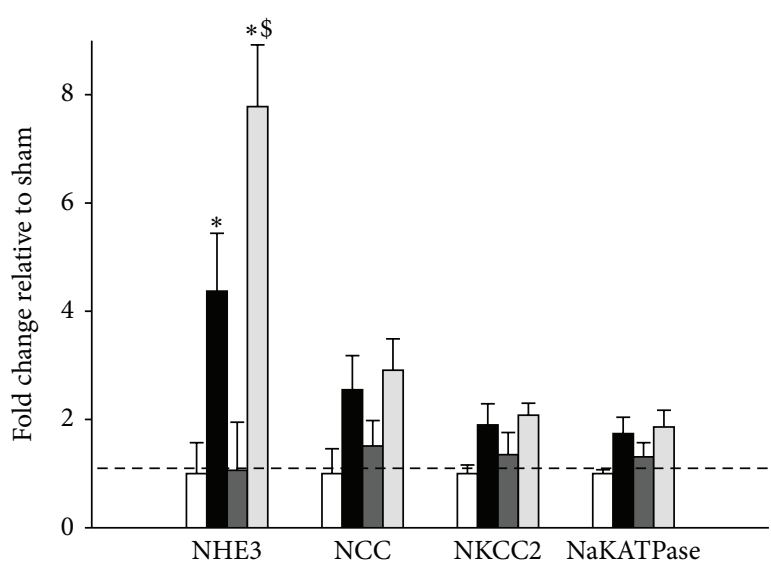

(b)

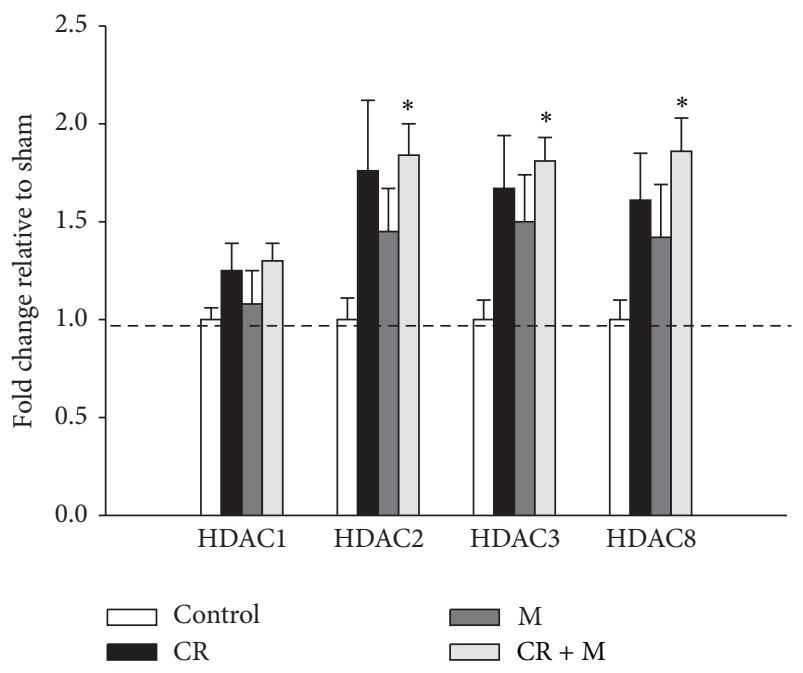

(d)

FIGURE 3: Effect of melatonin and CR on the expression of (a) apoptosis- and nephrogenesis-related genes, (b) sodium transporters, (c) reninangiotensin system (RAS) components, and (d) class I histone deacetylase (HDAC) in the kidney at 12 weeks of age. ${ }^{*} P<0.05$ versus control; ${ }^{\#} P<0.05$ versus $\mathrm{CR} ;{ }^{\$} \mathrm{P}<0.05$ versus $\mathrm{M}$.

We also determined whether melatonin regulated nephrogenesis-related genes, RAS components, sodium transporters, and HDACs during nephrogenesis. The mRNA levels in rat metanephroi grown in different concentrations of melatonin are shown in Figure 5. We found that low doses of melatonin had no effect on the expression of these genes, whereas high-dose melatonin treatment significantly increased expression of PAX2, renin, PRR, Mas, NHE3, and $\mathrm{Na}-\mathrm{K}-\mathrm{Cl}$ cotransporter 2 in metanephroi.

3.6. The Effects of Melatonin on Gene Expression during Nephrogenesis. We demonstrated that numerous individual genes were significantly regulated in the kidneys of offspring from melatonin-treated mothers during a critical period of renal development. As shown in Table 4, 439 and 15 genes were upregulated and downregulated, respectively. The most significantly regulated biological theme in the KEGG gene ontology analysis was tryptophan metabolism (Figure 6).

\section{Discussion}

The major findings of our study can be summarized as follows: (1) CR offspring developed hypertension at 12 weeks of age and this was prevented by maternal melatonin therapy; (2) melatonin restored the CR-induced increase of plasma ADMA level, decreased L-arginine level, and decreased Larginine-to-ADMA ratio; (3) CR reduced renal NO level and this was prevented by melatonin; (4) melatonin therapy increased PAX2 mRNA expression in the CR + M group; (5) CR upregulated renin and PRR expression and melatonin suppressed this increase; (6) melatonin therapy significantly increased renal ACE2 protein levels in the M and CR + M group; and (7) the expression of numerous genes was regulated in melatonin-treated offspring kidneys during nephrogenesis.

Our recent work indicates that ADMA-induced $\mathrm{NO} /$ reactive oxygen species (ROS) imbalance is involved in the development of hypertension in two different 


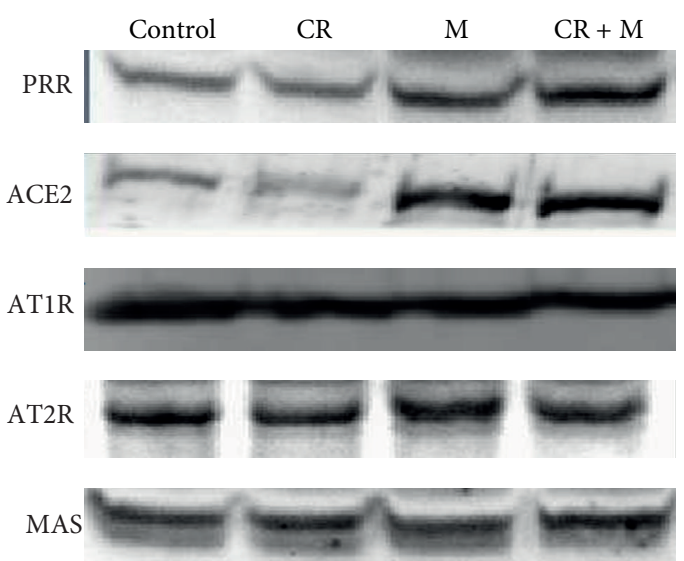

(a)

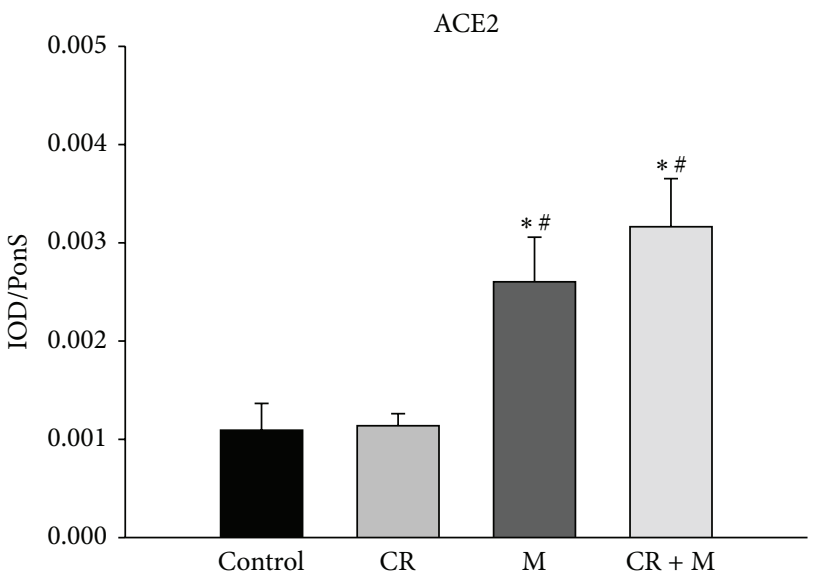

(c)

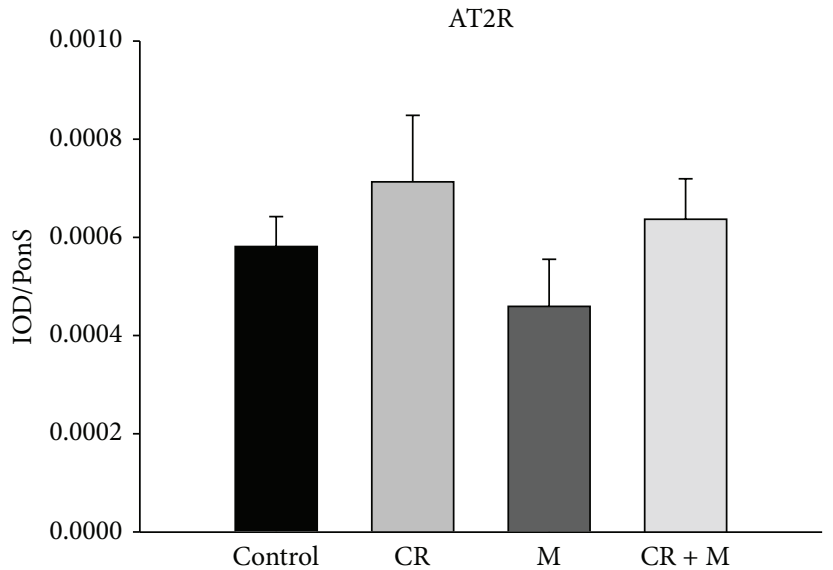

(e)

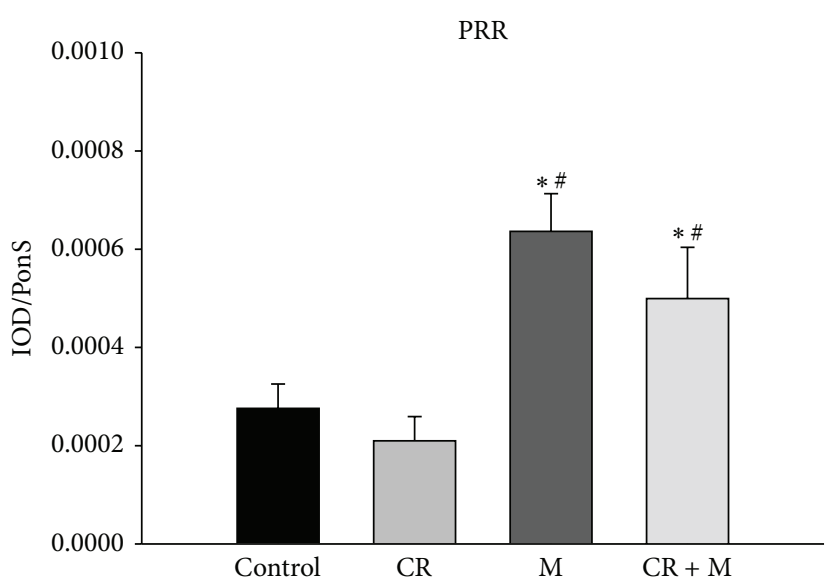

(b)

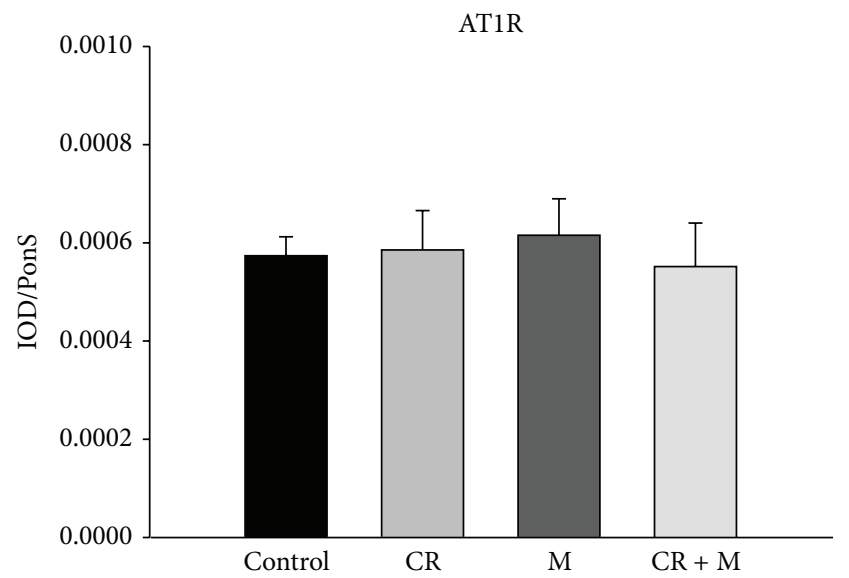

(d)

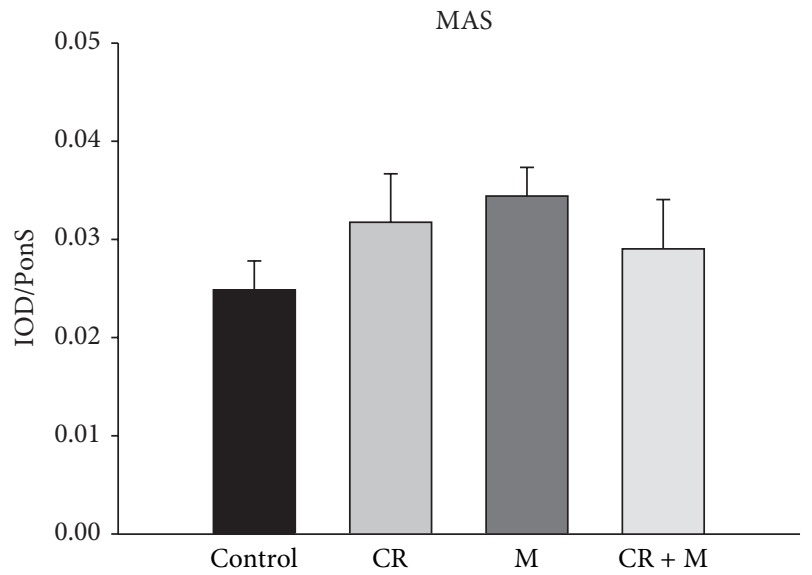

(f)

FIGURE 4: Representative western blots (a) showing prorenin receptor (PRR; $39 \mathrm{kDa})$, angiotensin-converting enzyme (ACE2; $50 \mathrm{kDa}$ ), angiotensin II type 1 (AT1R; $43 \mathrm{kDa}$ ) and type 2 (AT2R; $90 \mathrm{kDa}$ ), and MAS (37 kDa) proteins in male offspring kidneys at 12 weeks of age. Relative abundance of renal (b) PRR, (c) ACE2, (d) AT1R, (e) AT2R, and (f) MAS is quantified. ${ }^{*} P<0.05$ versus control; ${ }^{*} P<0.05$ versus CR.

developmental models, maternal caloric restriction, and maternal diabetes $[10,11]$. Several lines of evidence in this study indicated that ADMA-induced NO/ROS imbalance is involved in the developmental programming of hypertension in offspring exposed to maternal caloric restriction. First, plasma levels of the endogenous NOS inhibitor ADMA were increased in the CR group. Second, ADMA and L-arginine both compete for NOS and are present in a ratio that 


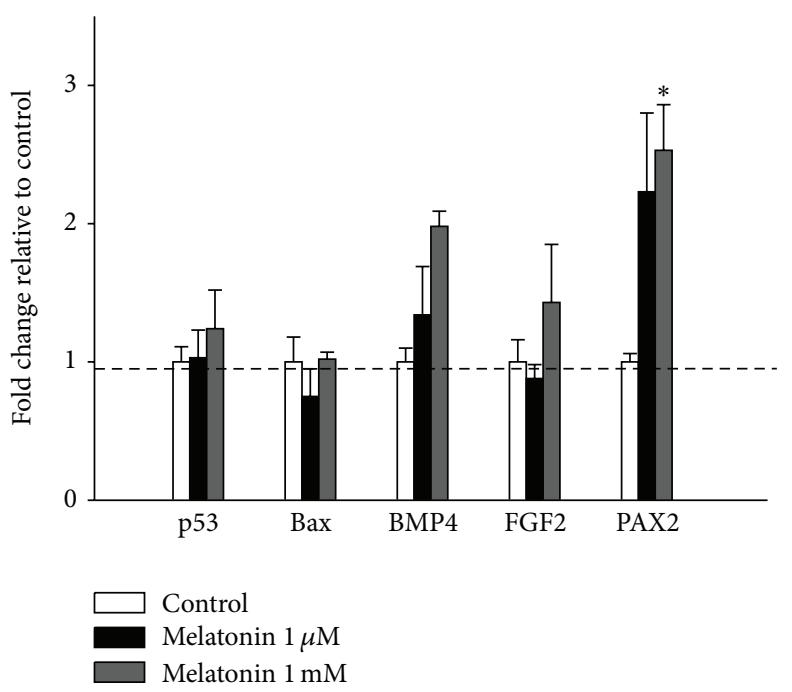

(a)

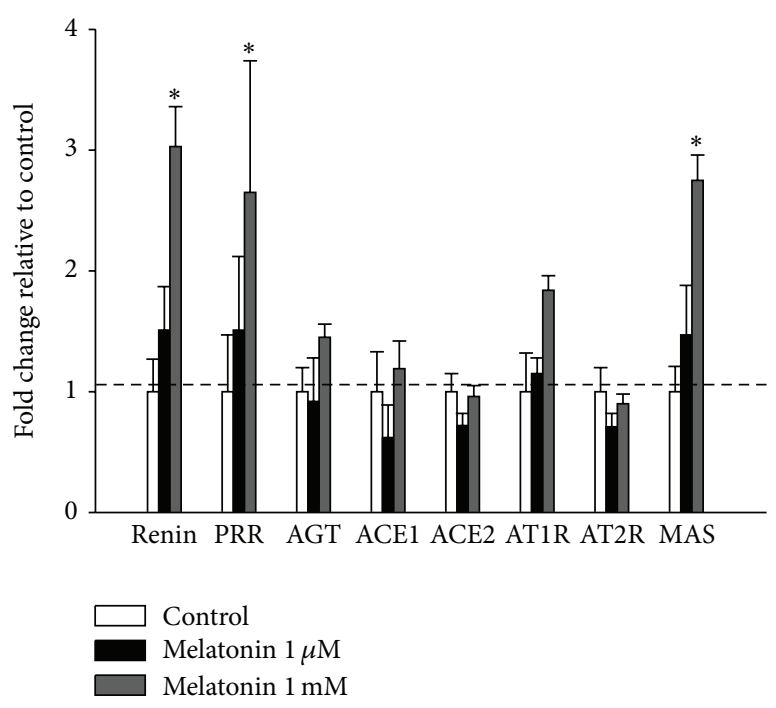

(b)

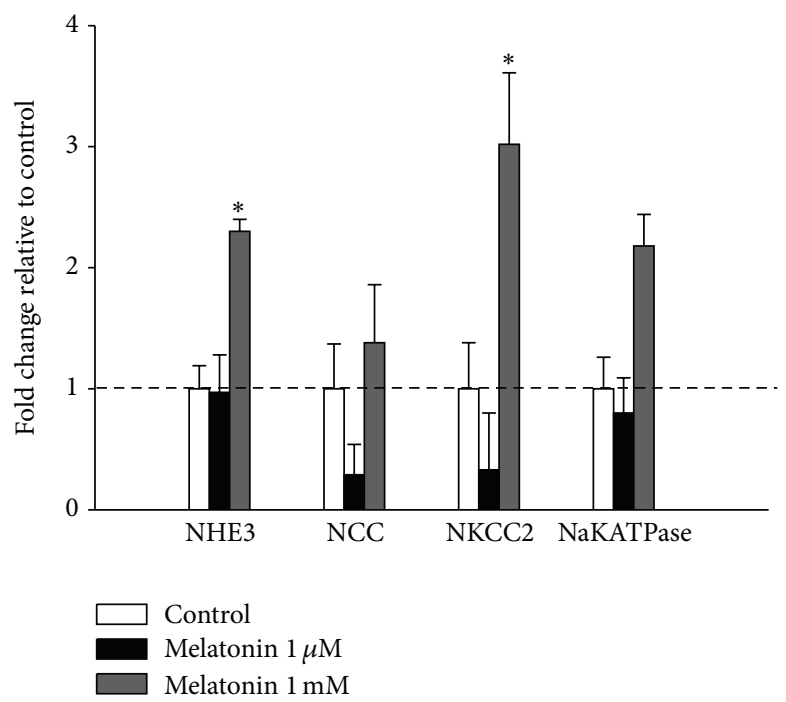

(c)

FIGURE 5: Gene expression of (a) apoptosis- and nephrogenesis-related genes, (b) RAS components, and (c) sodium transporters in the metanephroi of offspring from mothers treated with melatonin $(1 \mu \mathrm{M}$ or $1 \mathrm{mM}) .{ }^{*} P<0.05$ versus control $(n=5 /$ group$)$.

maintains NO homeostasis; this ratio was decreased in the plasma in the CR group. Third, maternal CR decreased renal NO levels in the offspring. Thus, alterations in the ADMA-NO pathway might be a major factor involved in programmed adult hypertension in response to maternal CR.

Melatonin is rapidly transferred from maternal to fetal circulation [20]. Administration of melatonin to pregnant rats prevents oxidative stress damage in the brains of offspring [21]. Previously, we showed that melatonin increases NO, restoring $\mathrm{NO} / \mathrm{ROS}$ balance at the prehypertension stage and leading to lower blood pressure in young SHR [15]. Consistent with these findings, we found that early melatonin therapy in the mother could prevent programmed hypertension in their adult offspring. Thus, we suggest that melatonin has a novel protective effect on programmed hypertension through acting on the ADMA-NO pathway.

In addition to oxidative stress, the RAS plays a fundamental role in the development of hypertension and kidney development [5]. Epigenetic regulation of several RAS components has been reported in different programmed hypertension models $[22,23]$. We demonstrated for the first time that melatonin therapy during nephrogenesis increased renin, PRR, and ACE2 expression in the kidney of the adult offspring. Consistent with these data, renal protein levels of PRR and ACE2 were increased in melatonin-treated offspring. Renin-PRR signaling is essential for proper kidney development and is causally linked to hypertension [13]. ACE2 appears to antagonize the effects of ACE through the production of angiotensin (1-7) in a manner that opposes 


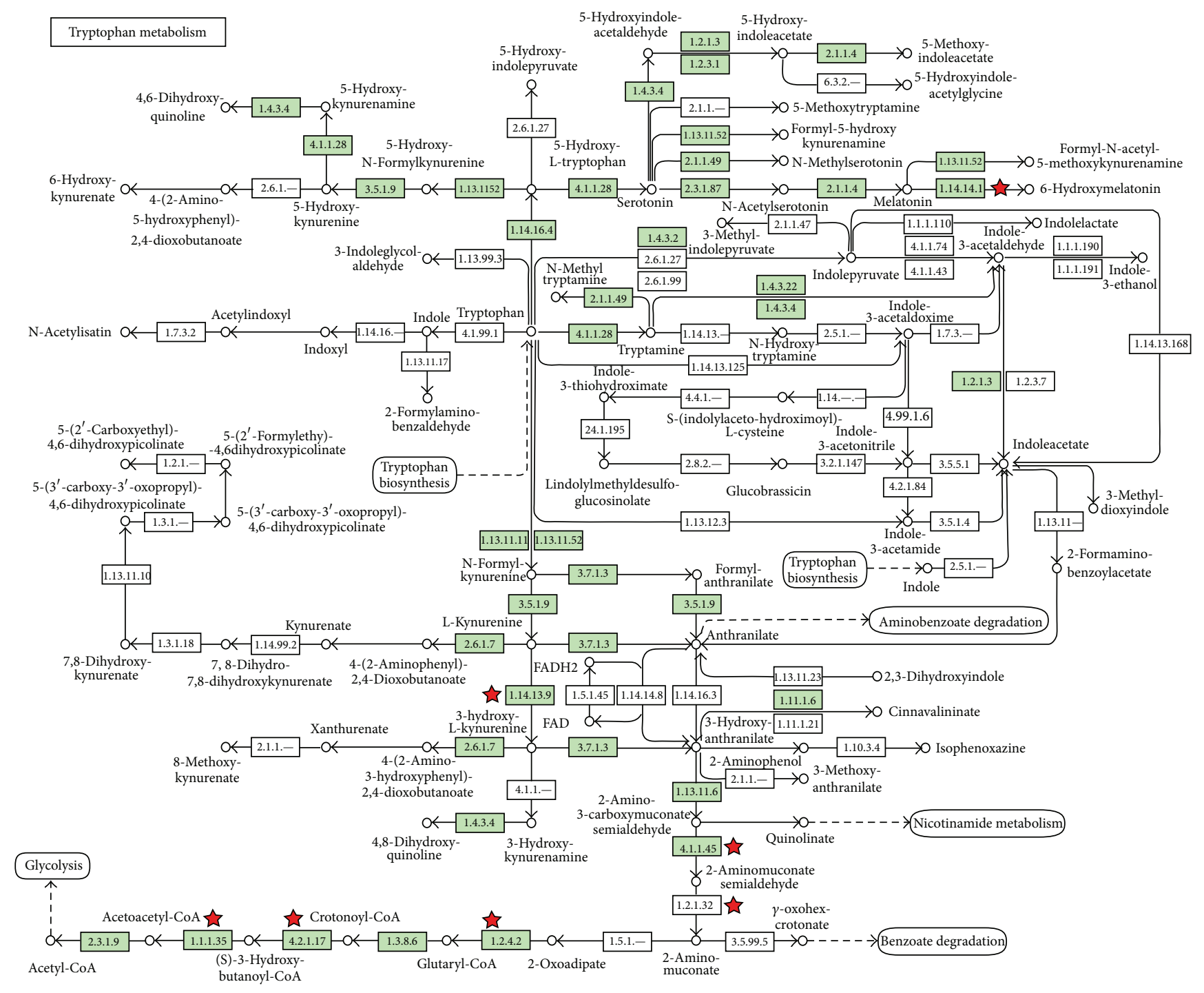

FIGURE 6: Enzymes of the tryptophan metabolism pathway that are regulated by melatonin therapy in the kidney (red stars). Data were analyzed using the KEGG Pathway feature of the DAVID software.

the development of hypertension [24]. Surprisingly, melatonin therapy increased ACE2 expression in the kidney and prevented CR-induced programmed hypertension, despite the presence of increased renin and PRR expression. Notably, melatonin upregulated several RAS components and had reciprocal effects on vasodilation and vasoconstriction in rats at 3 months of age. Future studies are required to clarify the underlying mechanisms involved in the differential regulation of RAS components by melatonin.

Long-term amelioration of hypertension by melatonin therapy during gestation and lactation may be due to epigenetic changes in the kidney during a critical period of nephrogenesis. We found that melatonin upregulated HDAC-2, -3, and -8 expression in the kidney in $\mathrm{CR}+\mathrm{M}$ group. This finding is consistent with that of our previous study showing that melatonin increased the expression of both class I and class II HDACs in vitro [25]. Given that melatonin increased class
I HDACs expression and that HDACs are primarily thought to repress gene transcription, melatonin likely upregulates gene expression. Conversely, melatonin is known as a class III HDAC inhibitor [17]. Thus, melatonin might have dual effects on HDACs to epigenetically regulate gene expression. To the best of our knowledge, our study is the first to document altered expression of more than 400 genes in the kidney in response to melatonin and implicates melatonin in the protection from programmed hypertension in adult life. Notably, our data imply that melatonin is liable to induce, but not suppress, gene expression in the developing kidney. Using the KEGG database, several biological pathways were proposed to be regulated by melatonin including focal adhesion signaling, the peroxisome proliferator-activated receptors signaling pathway, fatty acid metabolism, the transforming growth factor $\beta$ signaling pathway, and the Wnt signaling pathway. These findings suggest that melatonin might have 
TABLE 4: Genes that changed by RPKM > 0.3 in the kidney of melatonin treated offspring versus control at 1 week of age.

\begin{tabular}{|c|c|c|c|c|}
\hline Gene_ID & Gene symbol & Fold changes & $\log _{2}$ & $P$ value \\
\hline \multicolumn{5}{|c|}{ Upregulated: 439 genes } \\
\hline ENSRNOG00000038989 & D3ZSD6_RAT & 28.686 & 4.842 & 0.0083 \\
\hline ENSRNOG00000006367 & Slc5a8 & 19.264 & 4.268 & 0.0001 \\
\hline ENSRNOG00000003038 & $\mathrm{Sft} 2 \mathrm{~d} 2$ & 17.101 & 4.096 & 0.0020 \\
\hline ENSRNOG00000007720 & F1LX97_RAT & 13.841 & 3.791 & 0.0027 \\
\hline ENSRNOG00000019014 & Ndst1 & 12.657 & 3.662 & 0.0003 \\
\hline ENSRNOG00000017434 & Mgat3 & 12.364 & 3.628 & 0.0005 \\
\hline ENSRNOG00000001656 & Kcnj15 & 11.724 & 3.551 & 0.0028 \\
\hline ENSRNOG00000021292 & - & 11.449 & 3.517 & 0.0015 \\
\hline ENSRNOG00000017078 & Sepn1 & 11.107 & 3.473 & 0.0011 \\
\hline ENSRNOG00000030121 & Enpep & 10.970 & 3.456 & 0.0029 \\
\hline ENSRNOG00000005854 & Angptl & 10.920 & 3.449 & 0.0165 \\
\hline ENSRNOG00000009944 & LOC314407 & 10.858 & 3.441 & 0.0017 \\
\hline ENSRNOG00000013279 & Scd & 10.775 & 3.430 & 0.0012 \\
\hline ENSRNOG00000001724 & LOC678704 & 10.690 & 3.418 & 0.0011 \\
\hline ENSRNOG00000002463 & LOC682752 & 10.633 & 3.411 & 0.0033 \\
\hline ENSRNOG00000011630 & Ak3l1 & 10.304 & 3.365 & 0.0441 \\
\hline ENSRNOG00000005447 & RGD1311564 & 10.117 & 3.339 & 0.0026 \\
\hline ENSRNOG00000009019 & Slc6a6 & 10.090 & 3.335 & 0.0016 \\
\hline ENSRNOG00000002969 & Itpkb & 9.892 & 3.306 & 0.0020 \\
\hline ENSRNOG00000037307 & Spata22 & 9.876 & 3.304 & 0.0036 \\
\hline ENSRNOG00000039717 & Ipol1 & 9.584 & 3.261 & 0.0069 \\
\hline ENSRNOG00000025372 & Glce & 9.536 & 3.253 & 0.0023 \\
\hline ENSRNOG00000037884 & Oxgr1 & 9.510 & 3.249 & 0.0169 \\
\hline ENSRNOG00000021203 & Atl3 & 9.487 & 3.246 & 0.0056 \\
\hline ENSRNOG00000006787 & Dhcr 24 & 9.328 & 3.222 & 0.0023 \\
\hline ENSRNOG00000015038 & Adam10 & 9.279 & 3.214 & 0.0005 \\
\hline ENSRNOG00000002519 & Magtl & 9.253 & 3.210 & 0.0010 \\
\hline ENSRNOG00000038933 & D3ZF12_RAT & 9.225 & 3.206 & 0.0023 \\
\hline ENSRNOG00000024757 & RGD1310444 & 9.119 & 3.189 & 0.0066 \\
\hline ENSRNOG00000030285 & Epha3 & 9.064 & 3.180 & 0.0032 \\
\hline ENSRNOG00000018338 & Vwal & 9.017 & 3.173 & 0.0355 \\
\hline ENSRNOG00000022802 & Tmem184b & 8.982 & 3.167 & 0.0070 \\
\hline ENSRNOG00000013265 & Tgfbr2 & 8.947 & 3.161 & 0.0014 \\
\hline ENSRNOG00000026941 & Tril & 8.934 & 3.159 & 0.0024 \\
\hline ENSRNOG00000020532 & Kcnq1 & 8.904 & 3.154 & 0.0441 \\
\hline ENSRNOG00000018503 & LOC293190 & 8.862 & 3.148 & 0.0172 \\
\hline ENSRNOG00000002198 & LOC685352 & 8.711 & 3.123 & 0.0037 \\
\hline ENSRNOG00000017172 & Fam125b & 8.706 & 3.122 & 0.0291 \\
\hline ENSRNOG00000018554 & - & 8.663 & 3.115 & 0.0067 \\
\hline ENSRNOG00000013963 & IL6RB_RAT & 8.571 & 3.099 & 0.0023 \\
\hline ENSRNOG00000042565 & - & 8.547 & 3.095 & 0.0114 \\
\hline ENSRNOG00000032834 & Hspa13 & 8.544 & 3.095 & 0.0011 \\
\hline ENSRNOG00000002355 & Slc47al & 8.474 & 3.083 & 0.0025 \\
\hline ENSRNOG00000011927 & SDC3_RAT & 8.460 & 3.081 & 0.0065 \\
\hline ENSRNOG00000042540 & Mef2a & 8.454 & 3.080 & 0.0368 \\
\hline ENSRNOG00000029216 & Dgcr2 & 8.331 & 3.059 & 0.0175 \\
\hline ENSRNOG00000023725 & LOC689756 & 8.215 & 3.038 & 0.0191 \\
\hline ENSRNOG00000028129 & Fktn & 8.207 & 3.037 & 0.0063 \\
\hline ENSRNOG00000000547 & Tspyl4 & 8.202 & 3.036 & 0.0103 \\
\hline
\end{tabular}


TABLe 4: Continued.

\begin{tabular}{|c|c|c|c|c|}
\hline Gene_ID & Gene symbol & Fold changes & $\log _{2}$ & $P$ value \\
\hline ENSRNOG00000011859 & Eif5a2 & 8.192 & 3.034 & 0.0403 \\
\hline ENSRNOG00000028387 & E9PTK5_RAT & 8.168 & 3.030 & 0.0197 \\
\hline ENSRNOG00000015986 & Rassf8 & 8.134 & 3.024 & 0.0094 \\
\hline ENSRNOG00000029409 & Gstm6l & 8.062 & 3.011 & 0.0292 \\
\hline ENSRNOG00000008895 & Hnf4a & 7.993 & 2.999 & 0.0398 \\
\hline ENSRNOG00000038149 & Defb9 & 7.948 & 2.991 & 0.0437 \\
\hline ENSRNOG00000040287 & Cyp1b1 & 7.890 & 2.980 & 0.0439 \\
\hline ENSRNOG00000010468 & Elovl6 & 7.871 & 2.977 & 0.0394 \\
\hline ENSRNOG00000014524 & F1M9D3_RAT & 7.828 & 2.969 & 0.0077 \\
\hline ENSRNOG00000014209 & Utp6 & 7.793 & 2.962 & 0.0050 \\
\hline ENSRNOG00000013419 & Agphd1 & 7.789 & 2.961 & 0.0031 \\
\hline ENSRNOG00000020653 & S1pr2 & 7.775 & 2.959 & 0.0313 \\
\hline ENSRNOG00000018714 & Arl5b & 7.770 & 2.958 & 0.0078 \\
\hline ENSRNOG00000002408 & Rbm47 & 7.719 & 2.948 & 0.0057 \\
\hline ENSRNOG00000008971 & Hnf4g & 7.715 & 2.948 & 0.0085 \\
\hline ENSRNOG00000011271 & Mcc & 7.688 & 2.943 & 0.0120 \\
\hline ENSRNOG00000002276 & LOC100359714 & 7.641 & 2.934 & 0.0073 \\
\hline ENSRNOG00000009446 & Rxra & 7.607 & 2.927 & 0.0066 \\
\hline ENSRNOG00000019400 & Dag1 & 7.591 & 2.924 & 0.0010 \\
\hline ENSRNOG00000013098 & F1M9J1_RAT & 7.581 & 2.922 & 0.0320 \\
\hline ENSRNOG00000014511 & Alg10 & 7.551 & 2.917 & 0.0076 \\
\hline ENSRNOG00000012490 & Amph & 7.533 & 2.913 & 0.0461 \\
\hline ENSRNOG00000014934 & Fam63b & 7.481 & 2.903 & 0.0193 \\
\hline ENSRNOG00000039630 & LOC290577 & 7.414 & 2.890 & 0.0045 \\
\hline ENSRNOG00000032707 & Egf & 7.368 & 2.881 & 0.0017 \\
\hline ENSRNOG00000015605 & Ptprk & 7.357 & 2.879 & 0.0298 \\
\hline ENSRNOG00000000168 & Gatm & 7.311 & 2.870 & 0.0017 \\
\hline ENSRNOG00000027097 & F1M683_RAT & 7.273 & 2.863 & 0.0087 \\
\hline ENSRNOG00000018109 & Clic4 & 7.251 & 2.858 & 0.0048 \\
\hline ENSRNOG00000008629 & Secisbp2l & 7.236 & 2.855 & 0.0042 \\
\hline ENSRNOG00000019799 & Pcdhgc3 & 7.231 & 2.854 & 0.0246 \\
\hline ENSRNOG00000024089 & Fndc $3 b$ & 7.221 & 2.852 & 0.0065 \\
\hline ENSRNOG00000015852 & D4AD82_RAT & 7.192 & 2.846 & 0.0020 \\
\hline ENSRNOG00000006967 & Xiap & 7.151 & 2.838 & 0.0136 \\
\hline ENSRNOG00000031487 & F1LM52_RAT & 7.129 & 2.834 & 0.0477 \\
\hline ENSRNOG00000014866 & Pign & 7.077 & 2.823 & 0.0190 \\
\hline ENSRNOG00000033206 & Entpd5 & 7.060 & 2.820 & 0.0070 \\
\hline ENSRNOG00000037753 & Slc10a2 & 7.002 & 2.808 & 0.0089 \\
\hline ENSRNOG00000040195 & F1LZT0_RAT & 7.001 & 2.808 & 0.0018 \\
\hline ENSRNOG00000042817 & D4A5M8_RAT & 6.925 & 2.792 & 0.0104 \\
\hline ENSRNOG00000005070 & Spopl & 6.920 & 2.791 & 0.0139 \\
\hline ENSRNOG00000006459 & D4AEA4_RAT & 6.871 & 2.781 & 0.0251 \\
\hline ENSRNOG00000012784 & Gtf3c4 & 6.850 & 2.776 & 0.0096 \\
\hline ENSRNOG00000016968 & Gramd4 & 6.838 & 2.774 & 0.0216 \\
\hline ENSRNOG00000004448 & RGD1307051 & 6.819 & 2.770 & 0.0050 \\
\hline ENSRNOG00000021809 & Gpx3 & 6.801 & 2.766 & 0.0008 \\
\hline ENSRNOG00000014183 & Gnaq & 6.801 & 2.766 & 0.0084 \\
\hline ENSRNOG00000012991 & LOC100363275 & 6.798 & 2.765 & 0.0046 \\
\hline ENSRNOG00000013443 & $\operatorname{Tm} 9 \mathrm{sf3}$ & 6.791 & 2.764 & 0.0040 \\
\hline ENSRNOG00000042673 & LOC100359544 & 6.789 & 2.763 & 0.0012 \\
\hline
\end{tabular}


TABle 4: Continued.

\begin{tabular}{|c|c|c|c|c|}
\hline Gene_ID & Gene symbol & Fold changes & $\log _{2}$ & $P$ value \\
\hline ENSRNOG00000003873 & Cpd & 6.767 & 2.758 & 0.0028 \\
\hline ENSRNOG00000007990 & Adipor2 & 6.762 & 2.758 & 0.0026 \\
\hline ENSRNOG000000007804 & Clgalt1 & 6.762 & 2.757 & 0.0109 \\
\hline ENSRNOG00000043256 & D3ZNR8_RAT & 6.720 & 2.749 & 0.0145 \\
\hline ENSRNOG00000015124 & Gpam & 6.720 & 2.748 & 0.0109 \\
\hline ENSRNOG00000004888 & Spred2 & 6.690 & 2.742 & 0.0454 \\
\hline ENSRNOG00000003960 & Tmem27 & 6.682 & 2.740 & 0.0026 \\
\hline ENSRNOG00000015750 & Wnt7b & 6.654 & 2.734 & 0.0218 \\
\hline ENSRNOG00000030763 & Dpp4 & 6.601 & 2.723 & 0.0011 \\
\hline ENSRNOG000000039504 & Q5M885_RAT & 6.562 & 2.714 & 0.0116 \\
\hline ENSRNOG00000032768 & D3Z9G8_RAT & 6.497 & 2.700 & 0.0214 \\
\hline ENSRNOG00000039771 & LOC100361629 & 6.494 & 2.699 & 0.0140 \\
\hline ENSRNOG000000009274 & Futll & 6.475 & 2.695 & 0.0354 \\
\hline ENSRNOG00000027938 & RGD1562037 & 6.420 & 2.683 & 0.0117 \\
\hline ENSRNOG00000001335 & Zkscanl & 6.419 & 2.682 & 0.0077 \\
\hline ENSRNOG00000004978 & Prkacb & 6.379 & 2.673 & 0.0216 \\
\hline ENSRNOG00000005446 & Gnall & 6.363 & 2.670 & 0.0172 \\
\hline ENSRNOG000000003884 & Acmsd & 6.362 & 2.669 & 0.0262 \\
\hline ENSRNOG00000028190 & D4ACF8_RAT & 6.354 & 2.668 & 0.0432 \\
\hline ENSRNOG000000006338 & Lrp6 & 6.351 & 2.667 & 0.0041 \\
\hline ENSRNOG000000009523 & Rab1lfip2 & 6.345 & 2.666 & 0.0470 \\
\hline ENSRNOG000000003759 & Galc & 6.345 & 2.666 & 0.0140 \\
\hline ENSRNOG00000010620 & NDC1_RAT & 6.319 & 2.660 & 0.0275 \\
\hline ENSRNOG00000001821 & Adipoq & 6.306 & 2.657 & 0.0244 \\
\hline ENSRNOG000000038572 & RGD1562646 & 6.293 & 2.654 & 0.0106 \\
\hline ENSRNOG00000026120 & Fam8al & 6.282 & 2.651 & 0.0129 \\
\hline ENSRNOG00000025476 & RGD1359349 & 6.243 & 2.642 & 0.0126 \\
\hline ENSRNOG00000019508 & Wars2 & 6.216 & 2.636 & 0.0317 \\
\hline ENSRNOG00000008271 & Fam91a1 & 6.216 & 2.636 & 0.0031 \\
\hline ENSRNOG00000017120 & Abhd2 & 6.208 & 2.634 & 0.0278 \\
\hline ENSRNOG00000010843 & Nhlrc3 & 6.203 & 2.633 & 0.0255 \\
\hline ENSRNOG00000030704 & FILV74_RAT & 6.139 & 2.618 & 0.0369 \\
\hline ENSRNOG00000002509 & Gnl3l & 6.129 & 2.616 & 0.0124 \\
\hline ENSRNOG00000010841 & Col8a2 & 6.089 & 2.606 & 0.0457 \\
\hline ENSRNOG000000002728 & Btc & 6.088 & 2.606 & 0.0348 \\
\hline ENSRNOG00000027320 & Eif2cl & 6.082 & 2.605 & 0.0243 \\
\hline ENSRNOG00000009453 & Mobkl2b & 6.072 & 2.602 & 0.0271 \\
\hline ENSRNOG000000007797 & Rbpsuh & 6.069 & 2.602 & 0.0133 \\
\hline ENSRNOG00000017286 & HYES_RAT & 6.064 & 2.600 & 0.0032 \\
\hline ENSRNOG00000002461 & Nid1 & 6.057 & 2.599 & 0.0014 \\
\hline ENSRNOG00000006649 & Thrb & 6.048 & 2.596 & 0.0180 \\
\hline ENSRNOG00000025042 & Pde3a & 6.048 & 2.596 & 0.0189 \\
\hline ENSRNOG00000015916 & $\operatorname{Ttc} 38$ & 6.048 & 2.596 & 0.0384 \\
\hline ENSRNOG00000013581 & Extl3 & 6.038 & 2.594 & 0.0093 \\
\hline ENSRNOG00000002332 & MSPD1_RAT & 6.034 & 2.593 & 0.0213 \\
\hline ENSRNOG00000032757 & D3Z903_RAT & 6.032 & 2.593 & 0.0431 \\
\hline ENSRNOG000000029651 & Rdh2 & 6.025 & 2.591 & 0.0258 \\
\hline ENSRNOG00000018588 & Sox 4 & 6.019 & 2.590 & 0.0342 \\
\hline ENSRNOG00000012428 & Maf & 6.005 & 2.586 & 0.0483 \\
\hline ENSRNOG000000009506 & Mrella & 6.005 & 2.586 & 0.0332 \\
\hline ENSRNOG00000028330 & - & 5.987 & 2.582 & 0.0375 \\
\hline
\end{tabular}


TABle 4: Continued.

\begin{tabular}{|c|c|c|c|c|}
\hline Gene_ID & Gene symbol & Fold changes & $\log _{2}$ & $P$ value \\
\hline ENSRNOG00000034025 & D4A4T5_RAT & 5.979 & 2.580 & 0.0227 \\
\hline ENSRNOG00000007079 & Met & 5.979 & 2.580 & 0.0117 \\
\hline ENSRNOG00000008088 & Btbd3 & 5.979 & 2.580 & 0.0181 \\
\hline ENSRNOG00000017546 & Mylk3 & 5.945 & 2.572 & 0.0224 \\
\hline ENSRNOG00000042333 & Dnall & 5.895 & 2.559 & 0.0187 \\
\hline ENSRNOG00000001092 & $\mathrm{Kl}$ & 5.873 & 2.554 & 0.0106 \\
\hline ENSRNOG00000016498 & - & 5.836 & 2.545 & 0.0049 \\
\hline ENSRNOG00000037765 & Lims1 & 5.833 & 2.544 & 0.0367 \\
\hline ENSRNOG00000010267 & Klhdc10 & 5.827 & 2.543 & 0.0346 \\
\hline ENSRNOG00000043277 & D3ZIC7_RAT & 5.809 & 2.538 & 0.0206 \\
\hline ENSRNOG00000024799 & D3ZNV9_RAT & 5.803 & 2.537 & 0.0032 \\
\hline ENSRNOG00000004919 & Gns & 5.795 & 2.535 & 0.0282 \\
\hline ENSRNOG00000015080 & Wdfyl & 5.766 & 2.528 & 0.0292 \\
\hline ENSRNOG00000009565 & Pdk4 & 5.764 & 2.527 & 0.0206 \\
\hline ENSRNOG00000013082 & LCAP_RAT & 5.754 & 2.525 & 0.0322 \\
\hline ENSRNOG00000026501 & Slc6a19 & 5.742 & 2.522 & 0.0406 \\
\hline ENSRNOG00000009597 & Cyp4al & 5.740 & 2.521 & 0.0123 \\
\hline ENSRNOG00000011560 & Mtmr9 & 5.738 & 2.521 & 0.0368 \\
\hline ENSRNOG00000022710 & Prrg4 & 5.736 & 2.520 & 0.0269 \\
\hline ENSRNOG00000013469 & LOC100362805 & 5.715 & 2.515 & 0.0059 \\
\hline ENSRNOG00000024640 & RGD1304731 & 5.698 & 2.510 & 0.0080 \\
\hline ENSRNOG00000018952 & Sema3g & 5.692 & 2.509 & 0.0143 \\
\hline ENSRNOG00000020011 & Q66HF5_RAT & 5.677 & 2.505 & 0.0381 \\
\hline ENSRNOG00000012826 & Creb312 & 5.665 & 2.502 & 0.0189 \\
\hline ENSRNOG00000032492 & Usp22 & 5.657 & 2.500 & 0.0107 \\
\hline ENSRNOG00000021840 & LOC500046 & 5.644 & 2.497 & 0.0118 \\
\hline ENSRNOG00000034026 & Lclat1 & 5.642 & 2.496 & 0.0223 \\
\hline ENSRNOG00000009153 & Cidec & 5.642 & 2.496 & 0.0432 \\
\hline ENSRNOG00000028899 & Zbtb33 & 5.633 & 2.494 & 0.0168 \\
\hline ENSRNOG00000001766 & Tfrc & 5.613 & 2.489 & 0.0102 \\
\hline ENSRNOG00000017901 & Acy3 & 5.613 & 2.489 & 0.0044 \\
\hline ENSRNOG00000012095 & Pkia & 5.596 & 2.484 & 0.0339 \\
\hline ENSRNOG00000001796 & Dgkg & 5.573 & 2.479 & 0.0471 \\
\hline ENSRNOG00000004958 & RGD1304605 & 5.563 & 2.476 & 0.0100 \\
\hline ENSRNOG00000025587 & Plagl1 & 5.550 & 2.472 & 0.0289 \\
\hline ENSRNOG00000027540 & Fam102b & 5.536 & 2.469 & 0.0410 \\
\hline ENSRNOG00000001518 & Itga6 & 5.519 & 2.465 & 0.0452 \\
\hline ENSRNOG00000032723 & Eftudl & 5.515 & 2.463 & 0.0336 \\
\hline ENSRNOG00000002053 & F1M3H3_RAT & 5.491 & 2.457 & 0.0081 \\
\hline ENSRNOG00000003472 & Atp1lc-ps1 & 5.473 & 2.452 & 0.0317 \\
\hline ENSRNOG00000003984 & Apln & 5.448 & 2.446 & 0.0337 \\
\hline ENSRNOG00000012453 & RGD1564560 & 5.438 & 2.443 & 0.0046 \\
\hline ENSRNOG00000017846 & Slc44al & 5.422 & 2.439 & 0.0293 \\
\hline ENSRNOG00000016921 & Klhl11 & 5.418 & 2.438 & 0.0275 \\
\hline ENSRNOG00000026415 & D4A301_RAT & 5.403 & 2.434 & 0.0280 \\
\hline ENSRNOG00000013798 & Fnbp1l & 5.391 & 2.431 & 0.0098 \\
\hline ENSRNOG00000003620 & Fmo3 & 5.384 & 2.429 & 0.0050 \\
\hline ENSRNOG00000018220 & Pde4dip & 5.377 & 2.427 & 0.0462 \\
\hline ENSRNOG00000000145 & Pik3r3 & 5.352 & 2.420 & 0.0210 \\
\hline ENSRNOG00000008834 & LOC306096 & 5.351 & 2.420 & 0.0356 \\
\hline ENSRNOG00000025882 & Nipall & 5.345 & 2.418 & 0.0306 \\
\hline
\end{tabular}


TABle 4: Continued.

\begin{tabular}{|c|c|c|c|c|}
\hline Gene_ID & Gene symbol & Fold changes & $\log _{2}$ & $P$ value \\
\hline ENSRNOG00000010996 & Mobklla & 5.341 & 2.417 & 0.0147 \\
\hline ENSRNOG00000001582 & Bach1 & 5.339 & 2.417 & 0.0199 \\
\hline ENSRNOG00000022309 & D3ZRU8_RAT & 5.313 & 2.410 & 0.0048 \\
\hline ENSRNOG00000015741 & Slc2al3 & 5.298 & 2.406 & 0.0371 \\
\hline ENSRNOG00000014303 & F1M753_RAT & 5.294 & 2.404 & 0.0391 \\
\hline ENSRNOG00000036798 & Dusp3 & 5.284 & 2.402 & 0.0199 \\
\hline ENSRNOG00000012142 & Glyat & 5.283 & 2.401 & 0.0081 \\
\hline ENSRNOG00000024426 & D3ZXW1_RAT & 5.259 & 2.395 & 0.0477 \\
\hline ENSRNOG00000006628 & Dusp16 & 5.256 & 2.394 & 0.0271 \\
\hline ENSRNOG00000026143 & Ckap2l & 5.230 & 2.387 & 0.0271 \\
\hline ENSRNOG00000018867 & Klhdc7a & 5.223 & 2.385 & 0.0489 \\
\hline ENSRNOG00000025296 & Lrrc8a & 5.203 & 2.379 & 0.0176 \\
\hline ENSRNOG00000014508 & Mgll & 5.203 & 2.379 & 0.0137 \\
\hline ENSRNOG00000000589 & RGD1310495 & 5.199 & 2.378 & 0.0372 \\
\hline ENSRNOG00000014234 & Hiflan & 5.192 & 2.376 & 0.0394 \\
\hline ENSRNOG00000008450 & LOC100359539 & 5.178 & 2.372 & 0.0409 \\
\hline ENSRNOG00000010744 & Nrp1 & 5.177 & 2.372 & 0.0072 \\
\hline ENSRNOG00000039837 & RGD1563945 & 5.161 & 2.368 & 0.0466 \\
\hline ENSRNOG00000013177 & Map3k1 & 5.154 & 2.366 & 0.0114 \\
\hline ENSRNOG00000021719 & F1LX81_RAT & 5.153 & 2.365 & 0.0133 \\
\hline ENSRNOG000000024629 & Hadha & 5.116 & 2.355 & 0.0126 \\
\hline ENSRNOG00000014907 & Aldh8al & 5.105 & 2.352 & 0.0055 \\
\hline ENSRNOG00000036673 & Sectmlb & 5.098 & 2.350 & 0.0121 \\
\hline ENSRNOG00000024794 & Senp5 & 5.096 & 2.349 & 0.0264 \\
\hline ENSRNOG00000005131 & $\operatorname{Lin} 7 \mathrm{c}$ & 5.086 & 2.347 & 0.0289 \\
\hline ENSRNOG00000002225 & Scarb2 & 5.081 & 2.345 & 0.0116 \\
\hline ENSRNOG00000020284 & Prkar2a & 5.077 & 2.344 & 0.0215 \\
\hline ENSRNOG00000014648 & Efnb2 & 5.072 & 2.343 & 0.0303 \\
\hline ENSRNOG00000002488 & Galnt10 & 5.063 & 2.340 & 0.0437 \\
\hline ENSRNOG00000017406 & Atrnll & 5.056 & 2.338 & 0.0269 \\
\hline ENSRNOG00000010813 & Tspan14 & 5.048 & 2.336 & 0.0304 \\
\hline ENSRNOG00000000645 & Reep3 & 5.047 & 2.336 & 0.0262 \\
\hline ENSRNOG00000018873 & Fam168a & 5.036 & 2.332 & 0.0160 \\
\hline ENSRNOG00000020253 & RAB1B_RAT & 5.030 & 2.331 & 0.0128 \\
\hline ENSRNOG00000001235 & Gna12 & 5.012 & 2.325 & 0.0149 \\
\hline ENSRNOG00000040215 & F1LZL1_RAT & 5.011 & 2.325 & 0.0302 \\
\hline ENSRNOG00000011619 & Муо9а & 4.988 & 2.319 & 0.0163 \\
\hline ENSRNOG00000039976 & D3ZHG3_RAT & 4.983 & 2.317 & 0.0137 \\
\hline ENSRNOG00000016011 & Plekhg1 & 4.971 & 2.314 & 0.0315 \\
\hline ENSRNOG00000037909 & Ppmlf & 4.964 & 2.312 & 0.0269 \\
\hline ENSRNOG00000016419 & Pdlim5 & 4.962 & 2.311 & 0.0248 \\
\hline ENSRNOG00000023280 & Als2 & 4.952 & 2.308 & 0.0166 \\
\hline ENSRNOG00000005417 & Zhx2 & 4.948 & 2.307 & 0.0430 \\
\hline ENSRNOG00000017671 & Rasa3 & 4.944 & 2.306 & 0.0403 \\
\hline ENSRNOG00000016848 & Fzd4 & 4.942 & 2.305 & 0.0255 \\
\hline ENSRNOG00000003508 & LOC100364400 & 4.942 & 2.305 & 0.0244 \\
\hline ENSRNOG00000012394 & Bcl2113 & 4.931 & 2.302 & 0.0466 \\
\hline ENSRNOG00000018400 & D4AEL2_RAT & 4.931 & 2.302 & 0.0303 \\
\hline ENSRNOG00000013707 & Spatal3 & 4.930 & 2.302 & 0.0445 \\
\hline ENSRNOG00000002039 & LOC100360066 & 4.930 & 2.301 & 0.0436 \\
\hline ENSRNOG00000004563 & $\operatorname{Sec} 24 \mathrm{a}$ & 4.917 & 2.298 & 0.0191 \\
\hline
\end{tabular}


TABle 4: Continued.

\begin{tabular}{|c|c|c|c|c|}
\hline Gene_ID & Gene symbol & Fold changes & $\log _{2}$ & $P$ value \\
\hline ENSRNOG00000020386 & D3ZKH4_RAT & 4.906 & 2.295 & 0.0098 \\
\hline ENSRNOG00000007419 & Pank3 & 4.900 & 2.293 & 0.0128 \\
\hline ENSRNOG00000024533 & Aer61 & 4.889 & 2.290 & 0.0382 \\
\hline ENSRNOG00000027151 & Lrrc58 & 4.886 & 2.289 & 0.0393 \\
\hline ENSRNOG00000030124 & Ptpnl1 & 4.869 & 2.284 & 0.0160 \\
\hline ENSRNOG00000006131 & Mettl2 & 4.846 & 2.277 & 0.0271 \\
\hline ENSRNOG00000000407 & Dcbld1 & 4.834 & 2.273 & 0.0412 \\
\hline ENSRNOG00000008061 & Nuak1 & 4.826 & 2.271 & 0.0360 \\
\hline ENSRNOG00000037514 & Qser1 & 4.821 & 2.269 & 0.0136 \\
\hline ENSRNOG00000004959 & Actr2 & 4.807 & 2.265 & 0.0327 \\
\hline ENSRNOG00000028582 & F1M163_RAT & 4.795 & 2.261 & 0.0045 \\
\hline ENSRNOG00000043037 & LOC100366023 & 4.788 & 2.259 & 0.0349 \\
\hline ENSRNOG00000012135 & F1M2H7_RAT & 4.763 & 2.252 & 0.0406 \\
\hline ENSRNOG00000031069 & D4A9A7_RAT & 4.749 & 2.247 & 0.0462 \\
\hline ENSRNOG00000023109 & F1LVL2_RAT & 4.736 & 2.244 & 0.0482 \\
\hline ENSRNOG00000004442 & RGD1311756 & 4.729 & 2.241 & 0.0456 \\
\hline ENSRNOG00000021318 & Epas1 & 4.723 & 2.240 & 0.0138 \\
\hline ENSRNOG00000018099 & Itch & 4.702 & 2.233 & 0.0383 \\
\hline ENSRNOG00000038892 & LOC686123 & 4.691 & 2.230 & 0.0268 \\
\hline ENSRNOG00000000296 & Aqp6 & 4.685 & 2.228 & 0.0310 \\
\hline ENSRNOG00000014901 & Uggt1 & 4.684 & 2.228 & 0.0168 \\
\hline ENSRNOG00000019659 & Aspa & 4.680 & 2.227 & 0.0055 \\
\hline ENSRNOG00000010450 & D4ADY9_RAT & 4.662 & 2.221 & 0.0220 \\
\hline ENSRNOG00000011066 & 6-Mar & 4.658 & 2.220 & 0.0264 \\
\hline ENSRNOG00000013121 & Mier3 & 4.647 & 2.216 & 0.0408 \\
\hline ENSRNOG00000030894 & Slcola6 & 4.640 & 2.214 & 0.0068 \\
\hline ENSRNOG00000004964 & Erbb3 & 4.609 & 2.205 & 0.0351 \\
\hline ENSRNOG00000014135 & Rab11fip4 & 4.607 & 2.204 & 0.0453 \\
\hline ENSRNOG00000005052 & Slc39a9 & 4.594 & 2.200 & 0.0454 \\
\hline ENSRNOG00000005276 & Csnk2al & 4.589 & 2.198 & 0.0259 \\
\hline ENSRNOG00000015007 & RGD1565591 & 4.583 & 2.196 & 0.0462 \\
\hline ENSRNOG00000002099 & Wdfy3 & 4.579 & 2.195 & 0.0217 \\
\hline ENSRNOG00000001747 & Pak2 & 4.572 & 2.193 & 0.0178 \\
\hline ENSRNOG00000018226 & Zcchc14 & 4.565 & 2.190 & 0.0441 \\
\hline ENSRNOG00000010702 & Ube3c & 4.564 & 2.190 & 0.0154 \\
\hline ENSRNOG00000010610 & Hpgd & 4.556 & 2.188 & 0.0125 \\
\hline ENSRNOG00000001756 & D3ZDR3_RAT & 4.551 & 2.186 & 0.0486 \\
\hline ENSRNOG00000006335 & Klhl9 & 4.550 & 2.186 & 0.0083 \\
\hline ENSRNOG00000016715 & Kif11 & 4.547 & 2.185 & 0.0159 \\
\hline ENSRNOG00000021916 & Slc16a12 & 4.541 & 2.183 & 0.0224 \\
\hline ENSRNOG00000011250 & Inmt & 4.506 & 2.172 & 0.0125 \\
\hline ENSRNOG00000013140 & $\operatorname{Pdzd} 2$ & 4.502 & 2.171 & 0.0305 \\
\hline ENSRNOG00000012440 & Msra & 4.501 & 2.170 & 0.0308 \\
\hline ENSRNOG00000019932 & Ip6k1 & 4.500 & 2.170 & 0.0307 \\
\hline ENSRNOG00000037227 & Yes1 & 4.499 & 2.170 & 0.0412 \\
\hline ENSRNOG00000012054 & Zmpste24 & 4.498 & 2.169 & 0.0179 \\
\hline ENSRNOG00000007370 & Rnf144a & 4.493 & 2.168 & 0.0443 \\
\hline ENSRNOG00000022968 & F1M4Y9_RAT & 4.491 & 2.167 & 0.0400 \\
\hline ENSRNOG00000011340 & D3ZMJ4_RAT & 4.488 & 2.166 & 0.0143 \\
\hline ENSRNOG00000021705 & D3ZXN6_RAT & 4.486 & 2.165 & 0.0229 \\
\hline ENSRNOG00000003865 & Tmigd1 & 4.483 & 2.164 & 0.0072 \\
\hline
\end{tabular}


TABle 4: Continued.

\begin{tabular}{|c|c|c|c|c|}
\hline Gene_ID & Gene symbol & Fold changes & $\log _{2}$ & $P$ value \\
\hline $\begin{array}{l}\text { ENSRNOG00000012105 } \\
\text {. }\end{array}$ & F1MAE3_RAT & 4.478 & 2.163 & 0.0346 \\
\hline ENSRNOG00000011312 & F1LQ39_RAT & 4.475 & 2.162 & 0.0366 \\
\hline ENSRNOG00000000127 & F1LT58_RAT & 4.463 & 2.158 & 0.0484 \\
\hline ENSRNOG00000022929 & MTMRC_RAT & 4.438 & 2.150 & 0.0307 \\
\hline ENSRNOG00000033372 & Klhl24 & 4.431 & 2.148 & 0.0197 \\
\hline ENSRNOG00000008332 & Smo & 4.420 & 2.144 & 0.0209 \\
\hline ENSRNOG00000028616 & Pck1 & 4.418 & 2.143 & 0.0219 \\
\hline ENSRNOG00000013281 & Mibl & 4.415 & 2.142 & 0.0306 \\
\hline ENSRNOG00000011448 & Eril & 4.410 & 2.141 & 0.0414 \\
\hline ENSRNOG00000028422 & Rmnd5a & 4.409 & 2.141 & 0.0212 \\
\hline ENSRNOG00000014859 & Rnf152 & 4.404 & 2.139 & 0.0298 \\
\hline ENSRNOG00000001893 & LOC100362453 & 4.397 & 2.137 & 0.0349 \\
\hline ENSRNOG00000018123 & Ccny & 4.396 & 2.136 & 0.0173 \\
\hline ENSRNOG00000016337 & Slc22al & 4.394 & 2.135 & 0.0356 \\
\hline ENSRNOG00000003709 & Kmo & 4.389 & 2.134 & 0.0166 \\
\hline ENSRNOG00000019939 & CCND2_RAT & 4.386 & 2.133 & 0.0383 \\
\hline ENSRNOG00000029947 & - & 4.377 & 2.130 & 0.0399 \\
\hline ENSRNOG00000008346 & Itgb6 & 4.372 & 2.128 & 0.0245 \\
\hline ENSRNOG00000008678 & Antxr1 & 4.357 & 2.123 & 0.0237 \\
\hline ENSRNOG00000029924 & Klk1l & 4.344 & 2.119 & 0.0267 \\
\hline ENSRNOG00000043406 & LOC100360800 & 4.341 & 2.118 & 0.0323 \\
\hline ENSRNOG00000012343 & Pdp2 & 4.324 & 2.112 & 0.0419 \\
\hline ENSRNOG00000009899 & D3ZWL1_RAT & 4.306 & 2.106 & 0.0427 \\
\hline ENSRNOG00000003434 & Trove2 & 4.301 & 2.105 & 0.0368 \\
\hline ENSRNOG00000015519 & Cesld & 4.294 & 2.102 & 0.0253 \\
\hline ENSRNOG00000017439 & Cgnl1 & 4.294 & 2.102 & 0.0236 \\
\hline ENSRNOG00000014700 & $\operatorname{Ttc} 36$ & 4.287 & 2.100 & 0.0266 \\
\hline ENSRNOG00000007944 & Edem1 & 4.281 & 2.098 & 0.0367 \\
\hline ENSRNOG00000031263 & Haao & 4.246 & 2.086 & 0.0200 \\
\hline ENSRNOG00000001647 & Ets2 & 4.245 & 2.086 & 0.0357 \\
\hline ENSRNOG00000008652 & RGD1564964 & 4.226 & 2.079 & 0.0153 \\
\hline ENSRNOG00000023202 & Usp15 & 4.217 & 2.076 & 0.0230 \\
\hline ENSRNOG00000016289 & Bmprlb & 4.212 & 2.075 & 0.0370 \\
\hline ENSRNOG00000015024 & E9PT54_RAT & 4.208 & 2.073 & 0.0252 \\
\hline ENSRNOG00000000555 & Eif4ebp2 & 4.199 & 2.070 & 0.0381 \\
\hline ENSRNOG00000008620 & Smad3 & 4.198 & 2.070 & 0.0440 \\
\hline ENSRNOG00000008619 & Agtrap & 4.198 & 2.070 & 0.0217 \\
\hline ENSRNOG00000009711 & Hepacam2 & 4.196 & 2.069 & 0.0409 \\
\hline ENSRNOG00000015734 & Ube3a & 4.193 & 2.068 & 0.0225 \\
\hline ENSRNOG00000015634 & SMAD4_RAT & 4.189 & 2.067 & 0.0277 \\
\hline ENSRNOG00000042519 & RGD1312026 & 4.182 & 2.064 & 0.0380 \\
\hline ENSRNOG00000007564 & Evc & 4.160 & 2.057 & 0.0289 \\
\hline ENSRNOG00000008372 & Vamp7 & 4.160 & 2.057 & 0.0433 \\
\hline ENSRNOG00000024671 & D4AA13_RAT & 4.157 & 2.056 & 0.0120 \\
\hline ENSRNOG00000004622 & Calcrl & 4.142 & 2.050 & 0.0131 \\
\hline ENSRNOG00000009660 & Enpp6 & 4.140 & 2.050 & 0.0247 \\
\hline ENSRNOG00000014750 & D3ZXU7_RAT & 4.138 & 2.049 & 0.0176 \\
\hline ENSRNOG00000008694 & Miox & 4.134 & 2.048 & 0.0226 \\
\hline ENSRNOG00000004831 & Arid2 & 4.134 & 2.047 & 0.0317 \\
\hline ENSRNOG00000043167 & Itga9 & 4.124 & 2.044 & 0.0349 \\
\hline ENSRNOG00000001770 & Ehhadh & 4.114 & 2.040 & 0.0104 \\
\hline
\end{tabular}


TABle 4: Continued.

\begin{tabular}{|c|c|c|c|c|}
\hline Gene_ID & Gene symbol & Fold changes & $\log _{2}$ & $P$ value \\
\hline ENSRNOG00000042160 & Tmem167b & 4.112 & 2.040 & 0.0466 \\
\hline ENSRNOG00000018668 & Glg1 & 4.095 & 2.034 & 0.0172 \\
\hline ENSRNOG00000007985 & D4ABH6_RAT & 4.084 & 2.030 & 0.0231 \\
\hline ENSRNOG00000014623 & F1M3F2_RAT & 4.071 & 2.026 & 0.0332 \\
\hline ENSRNOG00000002227 & Kit & 4.056 & 2.020 & 0.0429 \\
\hline ENSRNOG00000016219 & Vnn1 & 4.052 & 2.019 & 0.0115 \\
\hline ENSRNOG00000008322 & E9PTI4_RAT & 4.035 & 2.013 & 0.0418 \\
\hline ENSRNOG00000011358 & Hipk3 & 4.034 & 2.012 & 0.0372 \\
\hline ENSRNOG00000028335 & Fat4 & 4.017 & 2.006 & 0.0190 \\
\hline ENSRNOG00000025554 & Zfp445 & 4.009 & 2.003 & 0.0365 \\
\hline ENSRNOG00000003388 & Cenpf & 3.990 & 1.996 & 0.0146 \\
\hline ENSRNOG00000000614 & Biccl & 3.987 & 1.995 & 0.0162 \\
\hline ENSRNOG00000039091 & D3ZRC4_RAT & 3.975 & 1.991 & 0.0145 \\
\hline ENSRNOG00000030154 & Сур4a2 & 3.962 & 1.986 & 0.0422 \\
\hline ENSRNOG00000033172 & - & 3.952 & 1.983 & 0.0176 \\
\hline ENSRNOG00000017466 & Kif5b & 3.949 & 1.981 & 0.0128 \\
\hline ENSRNOG00000042879 & D4A3X0_RAT & 3.943 & 1.979 & 0.0454 \\
\hline ENSRNOG00000002146 & $\operatorname{Pkd} 2$ & 3.942 & 1.979 & 0.0358 \\
\hline ENSRNOG00000012940 & Vps41 & 3.937 & 1.977 & 0.0280 \\
\hline ENSRNOG00000017291 & Sord & 3.928 & 1.974 & 0.0133 \\
\hline ENSRNOG00000001606 & Adamts5 & 3.923 & 1.972 & 0.0420 \\
\hline ENSRNOG00000016534 & D3ZKX0_RAT & 3.904 & 1.965 & 0.0295 \\
\hline ENSRNOG00000007202 & Sema3d & 3.898 & 1.963 & 0.0254 \\
\hline ENSRNOG00000012436 & Adh6 & 3.897 & 1.962 & 0.0137 \\
\hline ENSRNOG00000016334 & Rod1 & 3.867 & 1.951 & 0.0167 \\
\hline ENSRNOG00000018011 & RGD1564456 & 3.867 & 1.951 & 0.0417 \\
\hline ENSRNOG00000039494 & D4A608_RAT & 3.853 & 1.946 & 0.0328 \\
\hline ENSRNOG00000014976 & Acsm2 & 3.850 & 1.945 & 0.0330 \\
\hline ENSRNOG00000006636 & Otud6b & 3.817 & 1.932 & 0.0439 \\
\hline ENSRNOG00000015849 & Sepp1 & 3.812 & 1.930 & 0.0292 \\
\hline ENSRNOG00000004689 & Ptdss1 & 3.811 & 1.930 & 0.0342 \\
\hline ENSRNOG00000013808 & Ces2g & 3.803 & 1.927 & 0.0345 \\
\hline ENSRNOG00000014673 & Eri2 & 3.791 & 1.922 & 0.0429 \\
\hline ENSRNOG00000009819 & Vezf1 & 3.784 & 1.920 & 0.0450 \\
\hline ENSRNOG00000016758 & Loxl2 & 3.783 & 1.919 & 0.0310 \\
\hline ENSRNOG00000010061 & $\mathrm{Gmfb}$ & 3.763 & 1.912 & 0.0466 \\
\hline ENSRNOG00000023021 & Msl2 & 3.746 & 1.906 & 0.0486 \\
\hline ENSRNOG00000039571 & Glod5 & 3.742 & 1.904 & 0.0370 \\
\hline ENSRNOG00000017600 & Ptpn9 & 3.739 & 1.903 & 0.0329 \\
\hline ENSRNOG00000000590 & Naglt1 & 3.718 & 1.895 & 0.0365 \\
\hline ENSRNOG00000011511 & Stk24 & 3.716 & 1.894 & 0.0398 \\
\hline ENSRNOG00000018279 & Sfxn1 & 3.712 & 1.892 & 0.0213 \\
\hline ENSRNOG00000003953 & RB3GP_RAT & 3.709 & 1.891 & 0.0486 \\
\hline ENSRNOG00000024632 & Atf6 & 3.699 & 1.887 & 0.0421 \\
\hline ENSRNOG00000016779 & Fam120a & 3.679 & 1.879 & 0.0257 \\
\hline ENSRNOG00000010379 & Cugbpl & 3.670 & 1.876 & 0.0295 \\
\hline ENSRNOG00000010780 & Dlc1 & 3.664 & 1.874 & 0.0495 \\
\hline ENSRNOG00000003948 & Llgl1 & 3.659 & 1.871 & 0.0486 \\
\hline ENSRNOG00000016183 & Ipp & 3.647 & 1.867 & 0.0466 \\
\hline ENSRNOG00000017964 & Slc22a25 & 3.567 & 1.835 & 0.0201 \\
\hline ENSRNOG00000039745 & Pm20d1 & 3.557 & 1.831 & 0.0300 \\
\hline
\end{tabular}


TABLE 4: Continued.

\begin{tabular}{|c|c|c|c|c|}
\hline Gene_ID & Gene symbol & Fold changes & $\log _{2}$ & $P$ value \\
\hline ENSRNOG00000010107 & PALLD_RAT & 3.528 & 1.819 & 0.0467 \\
\hline ENSRNOG00000019444 & D4ADJ6_RAT & 3.515 & 1.814 & 0.0265 \\
\hline ENSRNOG00000011260 & $\mathrm{Cmbl}$ & 3.513 & 1.813 & 0.0221 \\
\hline ENSRNOG00000013322 & DPOLA_RAT & 3.505 & 1.810 & 0.0498 \\
\hline ENSRNOG00000039278 & Mcartl & 3.477 & 1.798 & 0.0345 \\
\hline ENSRNOG00000021108 & Slc22a12 & 3.449 & 1.786 & 0.0263 \\
\hline ENSRNOG00000010887 & RGD1309534 & 3.435 & 1.781 & 0.0382 \\
\hline ENSRNOG00000008331 & RGD1309995 & 3.394 & 1.763 & 0.0425 \\
\hline ENSRNOG00000007949 & Rgn & 3.355 & 1.746 & 0.0276 \\
\hline ENSRNOG00000011987 & Cd2ap & 3.343 & 1.741 & 0.0306 \\
\hline ENSRNOG00000042175 & B6VQA7_RAT & 3.331 & 1.736 & 0.0384 \\
\hline ENSRNOG00000012190 & Cldn2 & 3.324 & 1.733 & 0.0347 \\
\hline ENSRNOG00000023972 & F1M6Q3_RAT & 3.323 & 1.733 & 0.0322 \\
\hline ENSRNOG00000011763 & Serp1 & 3.319 & 1.731 & 0.0316 \\
\hline ENSRNOG00000004496 & Rock2 & 3.318 & 1.730 & 0.0337 \\
\hline ENSRNOG00000004677 & Zeb2 & 3.306 & 1.725 & 0.0306 \\
\hline ENSRNOG00000013409 & Gclm & 3.301 & 1.723 & 0.0338 \\
\hline ENSRNOG00000004302 & Pah & 3.270 & 1.709 & 0.0368 \\
\hline ENSRNOG00000010947 & MMP14_RAT & 3.253 & 1.702 & 0.0337 \\
\hline ENSRNOG00000011058 & Utrn & 3.247 & 1.699 & 0.0378 \\
\hline ENSRNOG00000018215 & Slc22a6 & 3.246 & 1.698 & 0.0377 \\
\hline ENSRNOG00000016456 & Il33 & 3.234 & 1.693 & 0.0319 \\
\hline ENSRNOG00000002541 & Pds5a & 3.164 & 1.662 & 0.0449 \\
\hline ENSRNOG00000002680 & Lamcl & 3.139 & 1.650 & 0.0366 \\
\hline ENSRNOG00000011124 & Eif4g2-ps1 & 3.125 & 1.644 & 0.0380 \\
\hline ENSRNOG00000004009 & Xpnpep2 & 3.118 & 1.641 & 0.0414 \\
\hline ENSRNOG00000010768 & Kpna4 & 3.114 & 1.639 & 0.0499 \\
\hline ENSRNOG00000042249 & F1LTA7_RAT & 3.101 & 1.633 & 0.0422 \\
\hline ENSRNOG00000014166 & Smoc2 & 3.078 & 1.622 & 0.0413 \\
\hline ENSRNOG00000002305 & Slc15a2 & 3.061 & 1.614 & 0.0400 \\
\hline ENSRNOG00000005130 & Ogdh & 3.049 & 1.608 & 0.0465 \\
\hline ENSRNOG00000018086 & Slc22a8 & 3.048 & 1.608 & 0.0418 \\
\hline ENSRNOG00000010814 & Bmprla & 3.006 & 1.588 & 0.0432 \\
\hline ENSRNOG00000032885 & CYC_RAT & 2.936 & 1.554 & 0.0478 \\
\hline \multicolumn{5}{|c|}{ Down-regulated Genes: 15} \\
\hline ENSRNOG00000032087 & F1LWC2_RAT & 0.301 & -1.734 & 0.0328 \\
\hline ENSRNOG00000032609 & - & 0.300 & -1.738 & 0.0394 \\
\hline ENSRNOG00000033748 & F1LWC2_RAT & 0.299 & -1.741 & 0.0381 \\
\hline ENSRNOG00000025670 & Shisa3 & 0.295 & -1.759 & 0.0282 \\
\hline ENSRNOG00000029115 & - & 0.284 & -1.815 & 0.0315 \\
\hline ENSRNOG00000011821 & S100a4 & 0.228 & -2.134 & 0.0086 \\
\hline ENSRNOG00000007632 & Zmynd17 & 0.211 & -2.243 & 0.0365 \\
\hline ENSRNOG00000025408 & D3ZTT0_RAT & 0.189 & -2.403 & 0.0422 \\
\hline ENSRNOG00000028844 & Slc9a5 & 0.181 & -2.466 & 0.0282 \\
\hline ENSRNOG00000006889 & Ambp & 0.150 & -2.741 & 0.0177 \\
\hline ENSRNOG00000028730 & D3ZI71_RAT & 0.148 & -2.757 & 0.0450 \\
\hline ENSRNOG00000026067 & Wfdc10 & 0.144 & -2.791 & 0.0123 \\
\hline ENSRNOG00000037374 & D3ZPQ1_RAT & 0.140 & -2.840 & 0.0087 \\
\hline ENSRNOG00000033517 & LOC100360791 & 0.129 & -2.950 & 0.0009 \\
\hline ENSRNOG00000042909 & F1LZX4_RAT & 0.107 & -3.229 & 0.0466 \\
\hline ENSRNOG00000014578 & Fxyd4 & 0.096 & -3.374 & 0.0001 \\
\hline
\end{tabular}


a global epigenetic effect during nephrogenesis. Interestingly, the most significantly regulated biological theme was tryptophan metabolism, indicating that melatonin might have a negative feedback effect on its precursor tryptophan. Notably, maternal melatonin therapy has adverse effects on survival and renal growth in Wistar-Kyoto rats [26]. Because our data showed that maternal melatonin therapy had strong epigenetic effects, further evaluation is warranted to determine whether early melatonin therapy causes long-term epigenetic changes that lead to adverse effects in adulthood.

Previously, we showed that maternal CR reduces nephron numbers in offspring [10]. Increases in renal apoptosis and impaired expression of nephrogenesis-related genes may contribute to this reduction. In contrast to several earlier reports $[27,28]$, we found that apoptosis- and nephrogenesisrelated genes were not altered in maternal CR-induced programmed hypertension. Of note, we showed for the first time that melatonin treatment upregulated PAX2 mRNA in metanephroi. Because PAX2 plays a crucial role in kidney development and is associated with various congenital renal and ureteral malformations, further studies are warranted to understand the epigenetic regulation of melatonin on PAX2 during nephrogenesis.

We conclude that prenatal melatonin therapy offsets the effects of maternal CR-induced programmed hypertension in adult offspring, primarily through the restoration of the ADMA-NO balance in the kidney. Our data suggested that a critical window exists during nephrogenesis in which the adult BP can be modified. Moreover, we showed that melatonin can modulate type I HDACs and serve as an inducer of gene expression in the developing kidney. The implications of melatonin-induced epigenetic changes on programmed hypertension in later life remain to be explored.

\section{Conflict of Interests}

The authors declare that there is no conflict of interests regarding the publication of this paper.

\section{Acknowledgments}

This work was supported by Grant NSC 101-2314-B-182A021-MY3 from the National Science Council (Taiwan) and Grants CMRPG8B0172 and CMRPG8C0041 from Chang Gung Memorial Hospital (Kaohsiung, Taiwan).

\section{References}

[1] N. B. Ojeda, D. Grigore, and B. T. Alexander, "Developmental programming of hypertension: insight from animal models of nutritional manipulation," Hypertension, vol. 52, no. 1, pp. 4450, 2008.

[2] S. P. Bagby, "Maternal nutrition, low nephron number, and hypertension in later life: pathways of nutritional programming," Journal of Nutrition, vol. 137, no. 4, pp. 1066-1072, 2007.

[3] I. C. McMillen and J. S. Robinson, "Developmental origins of the metabolic syndrome: prediction, plasticity, and programming," Physiological Reviews, vol. 85, no. 2, pp. 571-633, 2005.
[4] S. H. Alwasel and N. Ashton, "Prenatal programming of renal sodium handling in the rat," Clinical Science, vol. 117, no. 2, pp. 75-84, 2009.

[5] V. M. Vehaskari, T. Stewart, D. Lafont, C. Soyez, D. Seth, and J. Manning, "Kidney angiotensin and angiotensin receptor expression in prenatally programmed hypertension," The American Journal of Physiology-Renal Physiology, vol. 287, no. 2, pp. F262-F267, 2004.

[6] P. A. Dennery, "Oxidative stress in development: nature or nurture?" Free Radical Biology and Medicine, vol. 49, no. 7, pp. 1147-1151, 2010.

[7] C. S. Wilcox, "Oxidative stress and nitric oxide deficiency in the kidney: a critical link to hypertension?" The American Journal of Physiology-Regulatory Integrative and Comparative Physiology, vol. 289, no. 4, pp. R913-R935, 2005.

[8] C. Baylis, "Arginine, arginine analogs and nitric oxide production in chronic kidney disease," Nature Clinical Practice Nephrology, vol. 2, no. 4, pp. 209-220, 2006.

[9] Y.-L. Tain and L.-T. Huang, "Asymmetric dimethylarginine: clinical applications in pediatric medicine," Journal of the Formosan Medical Association, vol. 110, no. 2, pp. 70-77, 2011.

[10] Y.-L. Tain, C.-S. Hsieh, I.-C. Lin, C.-C. Chen, J.-M. Sheen, and L.-T. Huang, "Effects of maternal 1-citrulline supplementation on renal function and blood pressure in offspring exposed to maternal caloric restriction: the impact of nitric oxide pathway," Nitric Oxide, vol. 23, no. 1, pp. 34-41, 2010.

[11] Y. L. Tain, W. C. Lee, C. N. Hsu et al., "Asymmetric dimethylarginine is associated with developmental programming of adult kidney disease and hypertension in offspring of streptozotocintreated mothers," PLoS ONE, vol. 8, no. 2, Article ID e55420, 2013.

[12] S. Chen, C. Bellew, X. Yao et al., "Histone deacetylase (HDAC) activity is critical for embryonic kidney gene expression, growth, and differentiation," The Journal of Biological Chemistry, vol. 286, no. 37, pp. 32775-32789, 2011.

[13] R. Song, T. Van Buren, and I. V. Yosypiv, "Histone deacetylases are critical regulators of the renin-angiotensin system during ureteric bud branching morphogenesis," Pediatric Research, vol. 67, no. 6, pp. 573-578, 2010.

[14] A. Galano, D. X. Tan, and R. J. Reiter, "On the free radical scavenging activities of melatonin's metabolites, AFMK and AMK," Journal of Pineal Research, vol. 54, no. 3, pp. 245-257, 2013.

[15] Y.-L. Tain, L.-T. Huang, I.-C. Lin, Y.-T. Lau, and C.-Y. Lin, "Melatonin prevents hypertension and increased asymmetric dimethylarginine in young spontaneous hypertensive rats," Journal of Pineal Research, vol. 49, no. 4, pp. 390-398, 2010.

[16] A. Korkmaz, S. Rosales-Corral, and R. J. Reiter, "Gene regulation by melatonin linked to epigenetic phenomena," Gene, vol. 503, no. 1, pp. 1-11, 2012.

[17] B. Jung-Hynes, R. J. Reiter, and N. Ahmad, "Sirtuins, melatonin and circadian rhythms: building a bridge between aging and cancer," Journal of Pineal Research, vol. 48, no. 1, pp. 9-19, 2010.

[18] Y.-L. Tain and C. Baylis, "Determination of dimethylarginine dimethylaminohydrolase activity in the kidney," Kidney International, vol. 72, no. 7, pp. 886-889, 2007.

[19] A. Mortazavi, B. A. Williams, K. McCue, L. Schaeffer, and B. Wold, "Mapping and quantifying mammalian transcriptomes by RNA-Seq," Nature Methods, vol. 5, no. 7, pp. 621-628, 2008.

[20] Y. Okatani, A. Wakatsuki, and C. Kaneda, "Melatonin increases activities of glutathione peroxidase and superoxide dismutase 
in fetal rat brain," Journal of Pineal Research, vol. 28, no. 2, pp. 89-96, 2000.

[21] K. Watanabe, A. Wakatsuki, K. Shinohara, N. Ikenoue, K. Yokota, and T. Fukaya, "Maternally administered melatonin protects against ischemia and reperfusion-induced oxidative mitochondrial damage in premature fetal rat brain," Journal of Pineal Research, vol. 37, no. 4, pp. 276-280, 2004.

[22] I. Bogdarina, S. Welham, P. J. King, S. P. Burns, and A. J. L. Clark, "Epigenetic modification of the renin-angiotensin system in the fetal programming of hypertension," Circulation Research, vol. 100, no. 4, pp. 520-526, 2007.

[23] R. Goyal, D. Goyal, A. Leitzke, C. P. Gheorghe, and L. D. Longo, "Brain renin-angiotensin system: fetal epigenetic programming by maternal protein restriction during pregnancy," Reproductive Sciences, vol. 17, no. 3, pp. 227-238, 2010.

[24] Y. Feng, H. Xia, R. A. Santos, R. Speth, and E. Lazartigues, "Angiotensin-converting enzyme 2: a new target for neurogenic hypertension," Experimental Physiology, vol. 95, no. 5, pp. 601606, 2010.

[25] R. Sharma, T. Ottenhof, P. A. Rzeczkowska, and L. P. Niles, "Epigenetic targets for melatonin: induction of histone $\mathrm{H} 3$ hyperacetylation and gene expression in C17.2 neural stem cells," Journal of Pineal Research, vol. 45, no. 3, pp. 277-284, 2008.

[26] H. J. Singh, L. S. Keah, A. Kumar, and K. N. S. Sirajudeen, "Adverse effects of melatonin on rat pups of Wistar-Kyoto dams receiving melatonin supplementation during pregnancy," Experimental and Toxicologic Pathology, vol. 64, no. 7-8, pp. 751752, 2012.

[27] H. Dickinson, D. W. Walker, E. M. Wintour, and K. Moritz, "Maternal dexamethasone treatment at midgestation reduces nephron number and alters renal gene expression in the fetal spiny mouse," The American Journal of Physiology-Regulatory Integrative and Comparative Physiology, vol. 292, no. 1, pp. R453-R461, 2007.

[28] A. K. Abdel-Hakeem, T. Q. Henry, T. R. Magee et al., "Mechanisms of impaired nephrogenesis with fetal growth restriction: altered renal transcription and growth factor expression," The American Journal of Obstetrics and Gynecology, vol. 199, no. 3, pp. 252.e1-252.e7, 2008. 


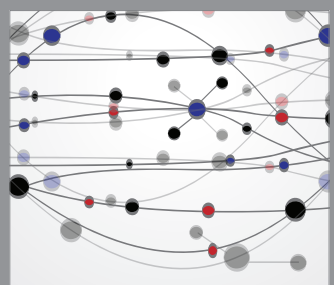

The Scientific World Journal
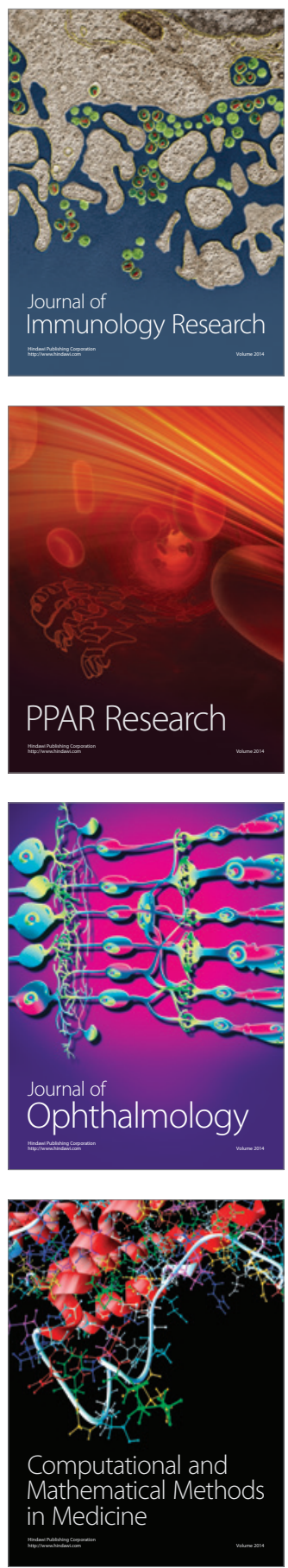

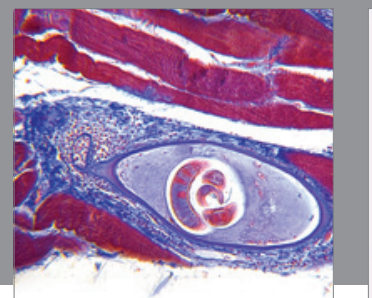

Gastroenterology

Research and Practice
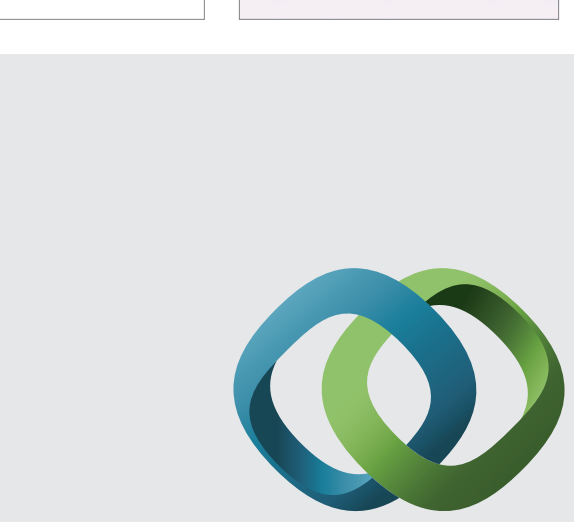

\section{Hindawi}

Submit your manuscripts at

http://www.hindawi.com
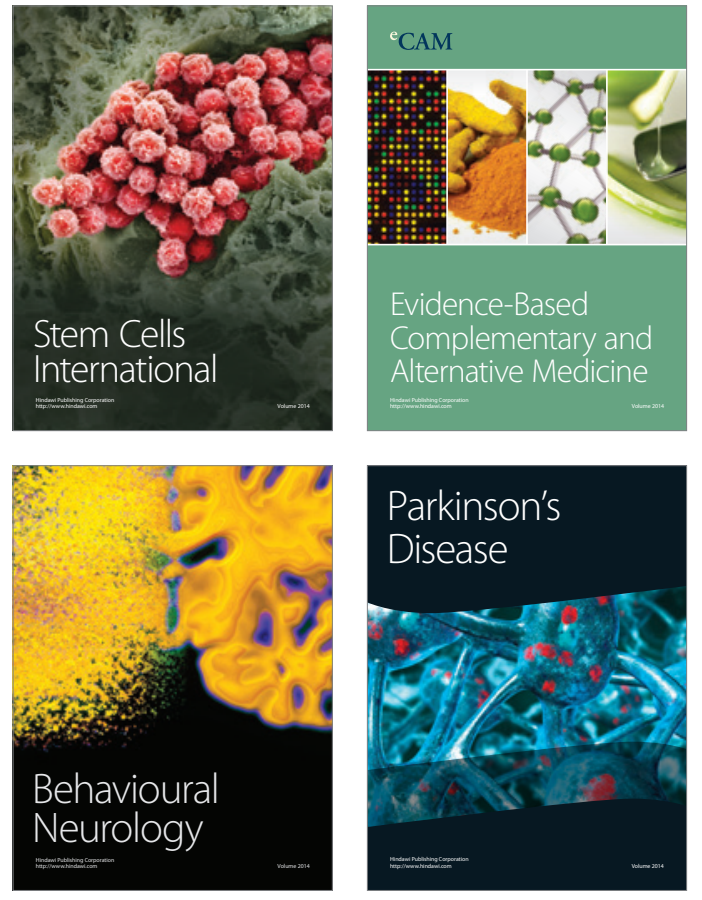
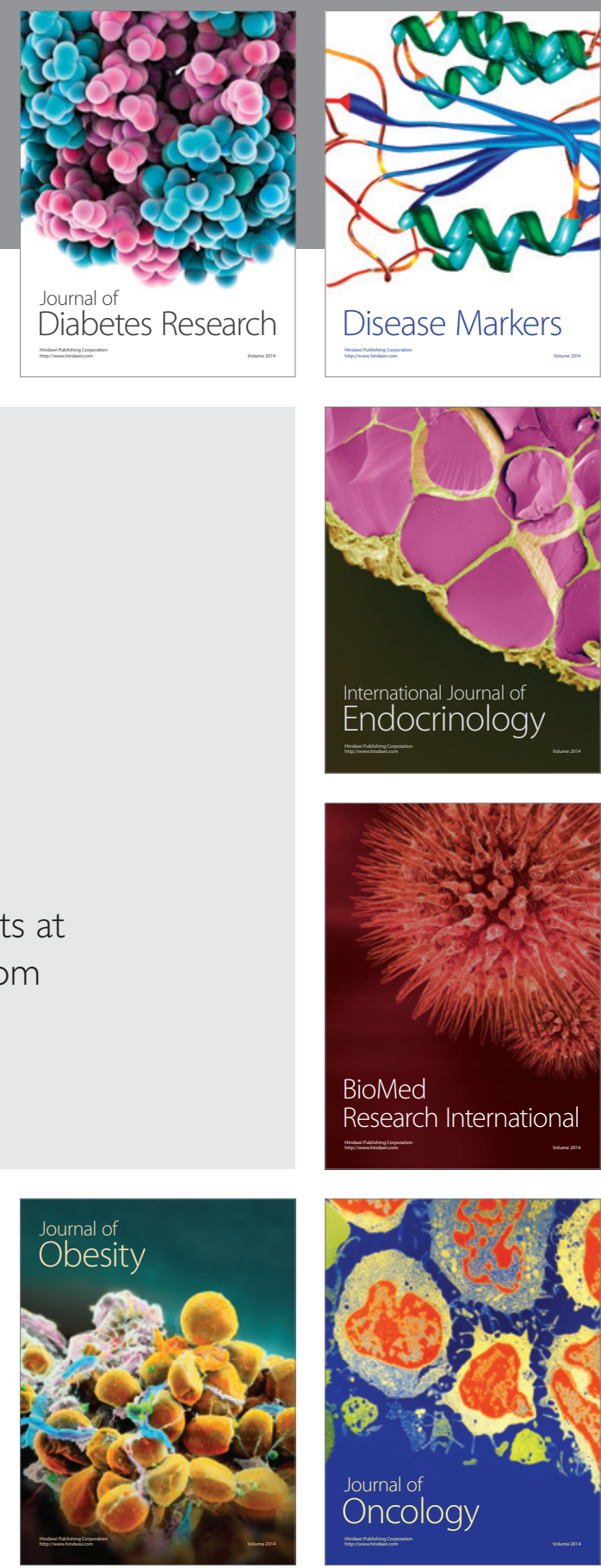

Disease Markers
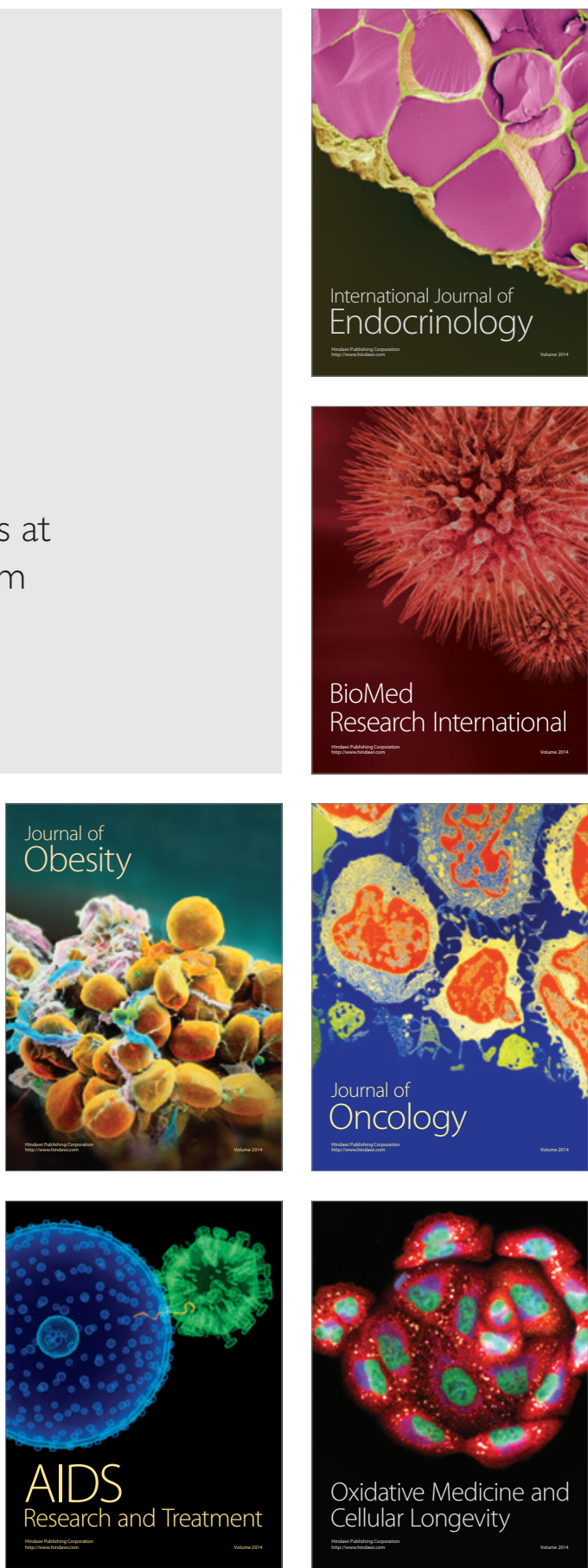